ESAIM: M2AN 48 (2014) 919-942

DOI: $10.1051 / \mathrm{m} 2 \mathrm{an} / 2013127$
ESAIM: Mathematical Modelling and Numerical Analysis

www.esaim-m2an.org

\title{
NUMERICAL ANALYSIS OF HISTORY-DEPENDENT QUASIVARIATIONAL INEQUALITIES WITH APPLICATIONS IN CONTACT MECHANICS
}

\author{
Kamran Kazmi ${ }^{1}$, Mikael Barboted ${ }^{2}$, Weimin Han $^{3}$ and Mircea Sofonea ${ }^{2}$
}

\begin{abstract}
A new class of history-dependent quasivariational inequalities was recently studied in [M. Sofonea and A. Matei, History-dependent quasivariational inequalities arising in contact mechanics. Eur. J. Appl. Math. 22 (2011) 471-491]. Existence, uniqueness and regularity results were proved and used in the study of several mathematical models which describe the contact between a deformable body and an obstacle. The aim of this paper is to provide numerical analysis of the quasivariational inequalities introduced in the aforementioned paper. To this end we introduce temporally semi-discrete and fully discrete schemes for the numerical approximation of the inequalities, show their unique solvability, and derive error estimates. We then apply these results to a quasistatic frictional contact problem in which the material's behavior is modeled with a viscoelastic constitutive law, the contact is bilateral, and friction is described with a slip-rate version of Coulomb's law. We discuss implementation of the corresponding fully-discrete scheme and present numerical simulation results on a two-dimensional example.
\end{abstract}

Mathematics Subject Classification. 65K15, 74D10, 74S05, 74S20.

Received July 25, 2012. Revised May 10, 2013.

Published online April 24, 2014.

\section{INTRODUCTION}

The theory of variational inequalities plays an important role in the study of both qualitative and numerical analysis of nonlinear boundary value problems arising in Mechanics, Physics and Engineering Science. For this reason, the mathematical literature dedicated to this field is extensive and the progress made in the last four decades is impressive. At the heart of this theory is the intrinsic inclusion of free boundaries in an elegant mathematical formulation. Existence and uniqueness results in the study of variational inequalities can be found in $[2,7,19,21,26,32]$. Details concerning numerical analysis of variational inequalities can be found in $[10,16,20]$. References in the study of mathematical and numerical analysis of variational inequalities arising in hardening plasticity include $[12,13]$.

Phenomena of contact between deformable bodies abound in industry and daily life. Contact of braking pads with wheels, tires with roads, pistons with skirts are just a few simple examples. Common industrial processes

\footnotetext{
Keywords and phrases. Quasivariational inequality, numerical analysis, finite element method, error estimates, quasistatic frictional contact problem, viscoelastic constitutive law, Coulomb's law, numerical simulations.

1 Department of Mathematics, University of Wisconsin Oshkosh, Oshkosh, WI 54901, USA. kazmis@uwosh.edu

2 Laboratoire de Mathématiques et Physique, University of Perpignan, 52 Avenue Paul Alduy, 66860 Perpignan, France. barboteu@univ-perp.fr; sofonea@univ-perp.fr

3 Department of Mathematics, University of Iowa, Iowa City, IA 52242, USA. weimin-han@uiowa.edu
} 
such as metal forming and metal extrusion involve contact evolution. Owing to their inherent complexity, contact phenomena lead to mathematical models expressed in terms of strongly nonlinear elliptic or evolutionary boundary value problems.

Considerable progress has been achieved recently in modeling, mathematical analysis and numerical simulations of various contact processes and, as a result, a general Mathematical Theory of Contact Mechanics is currently emerging. It is concerned with mathematical structures which underlie general contact problems with different materials, varied geometries and different contact conditions. Its aim is to provide a sound, clear and rigorous background to the construction of models for contact, to prove existence, uniqueness and regularity results, and to assign precise meaning to solutions, among others. Mathematical concepts involved include variational and hemivariational inequalities, and multivalued inclusions. An early attempt to study frictional contact problems within the framework of variational inequalities was made in [9]. A good reference on analysis and numerical approximations of contact problems involving elastic materials with or without friction is [20]. Variational analysis of various contact problems, including existence and uniqueness results, can be found in the monographs $[11,14,16,26,29,31]$. The state of the art in the field can also be found in the proceedings $[23,27,36]$ and in the special issue [28], as well.

In [33], new existence, uniqueness and regularity results are proved in the study of a class of quasivariational inequalities and are applied in the analysis of several quasistatic contact problems. This class of inequalities provides a general framework in which a large number of quasistatic contact problems, associated with various constitutive laws and frictional contact conditions, can be cast. Within particular settings of quasistatic process, in [33] it is shown how the models in Contact Mechanics lead to new types of variational inequalities and meanwhile, how abstract results on variational inequalities can be applied to prove the unique solvability of the corresponding contact problems.

The present paper represents a continuation of [33] and its aim is three folds. The first one is to provide numerical approximation of the abstract quasivariational inequalities studied in [33]. To this end we introduce temporally semi-discrete and fully discrete schemes, show their unique solvability, and derive error estimates. The second aim is to illustrate the use of the abstract results (concerning existence, uniqueness, regularity and approximation of the solution) in the study of a representative quasistatic frictional contact problem. The third aim is to test the performance of the fully discrete scheme on a frictional contact example.

The rest of the paper is structured as follows. In Section 2 we review some material from [33]. In Section 3 we study a temporally semi-discrete scheme for the quasivariational inequalities, and in Section 4 we study a fully discrete scheme. For both schemes we derive error estimates and prove convergence results. In Section 5 we introduce a quasistatic frictional contact problem in which the material's behavior is modeled with a viscoelastic constitutive law, the contact is bilateral and friction is described with a slip-rate dependent friction law. We show that this problem leads to a history-dependent quasivariational inequality for the velocity field. Then, in Section 6 we use our theoretical results in the variational and numerical analysis of this problem. Finally, in Section 7 we discuss implementation of the fully-discrete scheme and present numerical simulation results on a two-dimensional example.

We end this introduction by presenting some notation we shall use later in this paper. We use $\mathbb{N}^{*}$ for the set of positive integers and use $\mathbb{R}_{+}$for the set of non-negative real numbers, i.e. $\mathbb{R}_{+}=[0,+\infty)$. For a normed space $\left(Z,\|\cdot\|_{Z}\right)$ we use $C\left(\mathbb{R}_{+} ; Z\right)$ for the space of $Z$-valued continuous functions defined on $\mathbb{R}_{+}$and $C^{1}\left(\mathbb{R}_{+} ; Z\right)$ for the space of $Z$-valued continuously differentiable functions defined on $\mathbb{R}_{+}$. When $K \subset Z$, we use the notation $C\left(\mathbb{R}_{+} ; K\right)$ and $C^{1}\left(\mathbb{R}_{+} ; K\right)$ for the set of continuous and continuously differentiable functions defined on $\mathbb{R}_{+}$with values in $K$, respectively.

\section{ThE QUASIVARIATIONAL INEQUALITIES}

In this section we introduce the class of quasivariational inequalities we are interested in and recall the main results obtained in [33]. 
Let $X$ be a real Hilbert space with inner product $(\cdot, \cdot)_{X}$ and associated norm $\|\cdot\|_{X}, K \subset X$, and let $Y$ be a normed space with the norm $\|\cdot\|_{Y}$. Assumed given are operators $A: K \rightarrow X, \mathcal{S}: C\left(\mathbb{R}_{+} ; X\right) \rightarrow C\left(\mathbb{R}_{+} ; Y\right)$, functionals $\varphi: Y \times K \rightarrow \mathbb{R}, j: X \times K \rightarrow \mathbb{R}$, and a function $f: \mathbb{R}_{+} \rightarrow X$. Then, we consider the problem of finding a function $u \in C\left(\mathbb{R}_{+} ; X\right)$ such that for all $t \in \mathbb{R}_{+}$, the inequality below holds:

$$
\begin{aligned}
u(t) & \in K, \quad(A u(t), v-u(t))_{X}+\varphi(\mathcal{S} u(t), v)-\varphi(\mathcal{S} u(t), u(t)) \\
& +j(u(t), v)-j(u(t), u(t)) \geq(f(t), v-u(t))_{X} \quad \forall v \in K
\end{aligned}
$$

Note that (2.1) represents a time-dependent variational inequality governed by two functionals $\varphi$ and $j$ which depend on the solution and, therefore, we refer to (2.1) as a quasivariational inequality. To avoid any confusion, we note that here and below, $A u(t)$ and $\mathcal{S} u(t)$ are short hand notation for $A(u(t))$ and $(\mathcal{S} u)(t)$, i.e. $A u(t)=A(u(t))$ and $\mathcal{S} u(t)=(\mathcal{S} u)(t)$, for all $t \in \mathbb{R}_{+}$.

In the study of $(2.1)$ we assume that

$K$ is a closed, convex, nonempty subset of $X$

and $A: K \rightarrow X$ is a strongly monotone Lipschitz continuous operator, i.e.

$$
\left\{\begin{array}{l}
\text { (a) There exists } m>0 \text { such that } \\
\left(A u_{1}-A u_{2}, u_{1}-u_{2}\right)_{X} \geq m\left\|u_{1}-u_{2}\right\|_{X}^{2} \quad \forall u_{1}, u_{2} \in K . \\
\text { (b) There exists } L>0 \text { such that } \\
\left\|A u_{1}-A u_{2}\right\|_{X} \leq L\left\|u_{1}-u_{2}\right\|_{X} \quad \forall u_{1}, u_{2} \in K .
\end{array}\right.
$$

The functionals $\varphi: Y \times K \rightarrow \mathbb{R}$ and $j: X \times K \rightarrow \mathbb{R}$ satisfy

$$
\left\{\begin{array}{l}
\text { (a) For all } y \in Y, \varphi(y, \cdot) \text { is convex and lower semicontinuous on } K . \\
\text { (b) There exists } \alpha>0 \text { such that } \\
\varphi\left(y_{1}, u_{2}\right)-\varphi\left(y_{1}, u_{1}\right)+\varphi\left(y_{2}, u_{1}\right)-\varphi\left(y_{2}, u_{2}\right) \\
\leq \alpha\left\|y_{1}-y_{2}\right\|_{Y}\left\|u_{1}-u_{2}\right\|_{X} \quad \forall y_{1}, y_{2} \in Y, \forall u_{1}, u_{2} \in K .
\end{array}\right.
$$

(a) For all $x \in X, j(x, \cdot)$ is convex and lower semicontinuous on $K$.

(b) There exists $\beta>0$ such that

$$
\begin{aligned}
& j\left(u_{1}, v_{2}\right)-j\left(u_{1}, v_{1}\right)+j\left(u_{2}, v_{1}\right)-j\left(u_{2}, v_{2}\right) \\
& \leq \beta\left\|u_{1}-u_{2}\right\|_{X}\left\|v_{1}-v_{2}\right\|_{X} \quad \forall u_{1}, u_{2} \in X, \forall v_{1}, v_{2} \in K .
\end{aligned}
$$

Moreover, we assume that

$$
\beta<m,
$$

where $m$ and $\beta$ are the constants in (2.3) and (2.5), respectively. The operator $\mathcal{S}: C\left(\mathbb{R}_{+} ; X\right) \rightarrow C\left(\mathbb{R}_{+} ; Y\right)$ satisfies

$$
\left\{\begin{array}{l}
\text { For all } n \in \mathbb{N}^{*} \text { there exists } r_{n}>0 \text { such that } \\
\left\|\mathcal{S} u_{1}(t)-\mathcal{S} u_{2}(t)\right\|_{Y} \leq r_{n} \int_{0}^{t}\left\|u_{1}(s)-u_{2}(s)\right\|_{X} \mathrm{~d} s \\
\forall u_{1}, u_{2} \in C\left(\mathbb{R}_{+} ; X\right), \forall t \in[0, n]
\end{array}\right.
$$

and, finally, we assume that

$$
f \in C\left(\mathbb{R}_{+} ; X\right) .
$$


Note that condition (2.7) is satisfied for the operator $\mathcal{S}: C\left(\mathbb{R}_{+} ; X\right) \rightarrow C\left(\mathbb{R}_{+} ; Y\right)$ given by

$$
\mathcal{S} v(t)=R(t)\left(\int_{0}^{t} q(t, s) v(s) \mathrm{d} s+a\right) \quad \forall v \in C\left(\mathbb{R}_{+} ; X\right), \forall t \in \mathbb{R}_{+},
$$

where

$$
R \in C\left(\mathbb{R}_{+} ; \mathcal{L}(X, Y)\right), \quad q \in C\left(\mathbb{R}_{+} \times \mathbb{R}_{+} ; \mathcal{L}(X, X)\right), \quad a \in X,
$$

and $\mathcal{L}(X, Y)$ denotes the space of linear continuous operators from $X$ to $Y$ with the usual norm $\|\cdot\|_{\mathcal{L}(X, Y)}$. In particular, it is satisfied for the operator

$$
\mathcal{S} v(t)=R\left(\int_{0}^{t} v(s) \mathrm{d} s+a\right) \quad \forall v \in C\left(\mathbb{R}_{+} ; X\right), \forall t \in \mathbb{R}_{+},
$$

where $R \in \mathcal{L}(X, Y)$ and $a \in X$. Clearly, in the case of the operator (2.9) the current value $\mathcal{S} v(t)$ at the moment $t$ depends on the history of the values of $v(s)$ for the range $0 \leq s \leq t$ and, therefore, we refer the operators of this form as history-dependent operators. We extend this definition to all the operators $\mathcal{S}: C\left(\mathbb{R}_{+} ; X\right) \rightarrow$ $C\left(\mathbb{R}_{+} ; Y\right)$ which satisfy the condition (2.7) and, for this reason, we say that the quasivariational inequalities of the form (2.1) are history-dependent quasivariational inequalities. Their main feature is that at any moment $t \in \mathbb{R}_{+}$, the functional $\varphi$ depends on the history of the solution up to the moment $t, \mathcal{S} u(t)$. This feature distinguishes our quasivariational inequalities from those studied in the literature where $\varphi$ is usually assumed to depend only on the current value of the solution, $u(t)$.

The following result is proved in [33].

Theorem 2.1. Assume that (2.2)-(2.8) hold. Then, the variational inequality (2.1) has a unique solution $u \in$ $C\left(\mathbb{R}_{+} ; K\right)$.

The proof of Theorem 2.1 is based on arguments of monotonicity, convexity and fixed point. Its main ingredient is the use of a new fixed point result obtained in [30].

To describe the regularity of the solution of the variational inequality (2.1), we use below the standard notation for the spaces $C(I ; X), L^{p}(I ; X)$ and $W^{k, p}(I ; X)$, where $I \subset \mathbb{R}_{+}$is an interval, $1 \leq p \leq \infty, k=1,2, \ldots$, and the dot above represents the derivative with respect to the time variable. Also, we use the notation

$$
W_{\text {loc }}^{k, p}\left(\mathbb{R}_{+} ; X\right)=\left\{\boldsymbol{u}: \mathbb{R}_{+} \rightarrow X: \boldsymbol{u} \in W^{k, p}(I ; X) \quad \forall I \in \mathcal{C}_{+}\right\},
$$

where $\mathcal{C}_{+}$denotes the set of compact intervals included in $\mathbb{R}_{+}$. We use similar notation for a set of functions with values in $K$. Therefore, if $I, k, p$ and $\mathcal{C}_{+}$are as above, then $W^{k, p}(I ; K)$ denotes the set given by

$$
W^{k, p}(I ; K)=\left\{\boldsymbol{u}: \mathbb{R}_{+} \rightarrow K: \boldsymbol{u} \in W^{k, p}(I ; X)\right\},
$$

and $W_{\text {loc }}^{k, p}\left(\mathbb{R}_{+} ; K\right)$ represents the set

$$
W_{\text {loc }}^{k, p}\left(\mathbb{R}_{+} ; K\right)=\left\{\boldsymbol{u}: \mathbb{R}_{+} \rightarrow K: \boldsymbol{u} \in W^{k, p}(I ; K) \quad \forall I \in \mathcal{C}_{+}\right\} .
$$

The following regularity result is obtained in [33].

Theorem 2.2. Assume that (2.2)-(2.8) hold and, moreover, assume that $Y$ is a reflexive Banach space. Assume in addition that there exists $p \in[1, \infty]$ such that

$$
\begin{aligned}
f & \in W_{\text {loc }}^{1, p}\left(\mathbb{R}_{+} ; X\right), \\
\mathcal{S} v & \in W_{\text {loc }}^{1, p}\left(\mathbb{R}_{+} ; Y\right) \quad \forall v \in C\left(\mathbb{R}_{+} ; X\right) .
\end{aligned}
$$

Then, the solution of the variational inequality (2.1) obtained in Theorem 2.1 has the regularity $u \in$ $W_{\text {loc }}^{1, p}\left(\mathbb{R}_{+} ; K\right)$. 


\section{TEMPORALLY SEMI-DISCRETE APPROXIMATION}

In the next two sections, we provide the numerical solution of problem (2.1) and derive some error estimates. For computational purposes, we consider the problem (2.1) on a finite time interval $I=[0, T]$ where $T$ is arbitrary but fixed. That is, we consider the following problem of finding a function $u \in C(I ; X)$ such that for all $t \in I$,

$$
\begin{aligned}
u(t) & \in K, \quad(A u(t), v-u(t))_{X}+\varphi(\mathcal{S} u(t), v)-\varphi(\mathcal{S} u(t), u(t)) \\
& +j(u(t), v)-j(u(t), u(t)) \geq(f(t), v-u(t))_{X} \quad \forall v \in K .
\end{aligned}
$$

In this section we study a semi-discrete scheme for the problem (3.1) where the time variable is discretized. We consider $\mathcal{S}$ in the form (2.9) and we assume that (2.2)-(2.6), (2.8) and (2.10) hold.

Let $0=t_{0}<t_{1}<\cdots<t_{N}=T$ be a uniform partition of the time interval $[0, T]$, i.e., $t_{n}=n k, 0 \leq n \leq N$, $k=T / N$. For a continuous function $v(t)$ with values in a function space, we write $v_{j}=v\left(t_{j}\right), 0 \leq j \leq N$. We take the trapezoidal rule as an example for the discretization of the time integration. All the discussion and results below can be extended to schemes based on other numerical integration formulas. Recall that the trapezoidal rule is

$$
\int_{0}^{t_{n}} z(s) \mathrm{d} s \approx k \sum_{j=0}^{n} z\left(t_{j}\right),
$$

where a prime indicates that the first and last terms in the summation are to be halved. Corresponding to the operator $\mathcal{S}$ of $(2.9)$, we introduce its approximation

$$
\mathcal{S}_{n}^{k} v=R\left(t_{n}\right)\left(k \sum_{j=0}^{n} q\left(t_{n}, t_{j}\right) v_{j}+a\right) .
$$

Then the temporally semi-discrete scheme for the problem (3.1) is to find the discrete solution $u^{k}:=\left\{u_{n}^{k}\right\}_{n=0}^{N} \subset$ $K$ such that

$$
\left(A u_{n}^{k}, v-u_{n}^{k}\right)_{X}+\varphi\left(\mathcal{S}_{n}^{k} u^{k}, v\right)-\varphi\left(\mathcal{S}_{n}^{k} u^{k}, u_{n}^{k}\right)+j\left(u_{n}^{k}, v\right)-j\left(u_{n}^{k}, u_{n}^{k}\right) \geq\left(f_{n}, v-u_{n}^{k}\right)_{X} \quad \forall v \in K .
$$

We consider the constant $c_{1}$, independent of $k$ and $N$, given by

$$
c_{1}=\|R\|_{C(I ; \mathcal{L}(X, Y))}\|q\|_{C(I \times I ; \mathcal{L}(X, X))} .
$$

Then, we use (2.6) to consider the smallness condition

$$
k<\frac{m-\beta}{\alpha c_{1}} .
$$

We have the following existence and uniqueness result.

Theorem 3.1. Assume that (2.2)-(2.6) and (2.8)-(2.10) hold. Then the problem (3.4) has a unique solution for all $k$ satisfying (3.6).

Proof. With $\left\{u_{j}^{k}\right\}_{j \leq n-1} \subset K$ known, we show that (3.4) uniquely determines $u_{n}^{k} \in K$. The proof is divided into several steps. In the first step, let $\eta \in X$ be given. Denote

$$
u^{k \eta}=\left\{u_{0}^{k}, \ldots, u_{n-1}^{k}, \eta\right\},
$$


and

$$
y_{\eta}=\mathcal{S}_{n}^{k} u^{k \eta} .
$$

Consider now the auxiliary problem of finding $u_{\eta} \in K$ such that

$$
\left(A u_{\eta}, v-u_{\eta}\right)_{X}+\varphi\left(y_{\eta}, v\right)-\varphi\left(y_{\eta}, u_{\eta}\right)+j(\eta, v)-j\left(\eta, u_{\eta}\right) \geq\left(f_{n}, v-u_{\eta}\right)_{X} \quad \forall v \in K .
$$

Under the assumptions (2.2)-(2.5), it follows from the classical results for elliptic variational inequalities (see e.g. [14]) that there exists a unique solution $u_{\eta} \in K$ of the problem (3.8).

In the next step, we define an operator $\Lambda: X \rightarrow K$ by

$$
\Lambda \eta=u_{\eta},
$$

and show that the operator $\Lambda$ has a unique fixed point $\eta^{*} \in K$. Let $\eta_{1}$ and $\eta_{2} \in X$ be given and denote $y_{i}=y_{\eta_{i}}$ and $u_{i}=u_{\eta_{i}}, i=1,2$. Then $u_{1}$ and $u_{2} \in K$ satisfy

$$
\begin{array}{ll}
\left(A u_{1}, v-u_{1}\right)_{X}+\varphi\left(y_{1}, v\right)-\varphi\left(y_{1}, u_{1}\right)+j\left(\eta_{1}, v\right)-j\left(\eta_{1}, u_{1}\right) \geq\left(f_{n}, v-u_{1}\right)_{X} & \forall v \in K, \\
\left(A u_{2}, v-u_{2}\right)_{X}+\varphi\left(y_{2}, v\right)-\varphi\left(y_{2}, u_{2}\right)+j\left(\eta_{2}, v\right)-j\left(\eta_{2}, u_{2}\right) \geq\left(f_{n}, v-u_{2}\right)_{X} & \forall v \in K .
\end{array}
$$

Now we take $v=u_{2}$ in (3.10) and $v=u_{1}$ in (3.11) and add the resulting inequalities to obtain

$$
\begin{aligned}
\left(A u_{1}-A u_{2}, u_{1}-u_{2}\right)_{X} \leq & \varphi\left(y_{1}, u_{2}\right)-\varphi\left(y_{1}, u_{1}\right)+\varphi\left(y_{2}, u_{1}\right)-\varphi\left(y_{2}, u_{2}\right) \\
& +j\left(\eta_{1}, u_{2}\right)-j\left(\eta_{1}, u_{1}\right)+j\left(\eta_{2}, u_{1}\right)-j\left(\eta_{2}, u_{2}\right) .
\end{aligned}
$$

Then we use assumptions $(2.3)(\mathrm{a}),(2.4)(\mathrm{b})$, and $(2.5)(\mathrm{b})$ to get

$$
m\left\|u_{1}-u_{2}\right\|_{X} \leq \alpha\left\|y_{1}-y_{2}\right\|_{Y}+\beta\left\|\eta_{1}-\eta_{2}\right\|_{X} .
$$

By the definition (3.9) of the operator $\Lambda$, we have

$$
\left\|\Lambda \eta_{1}-\Lambda \eta_{2}\right\|_{X}=\left\|u_{1}-u_{2}\right\|_{X} .
$$

Therefore, from (3.12) we obtain

$$
\left\|\Lambda \eta_{1}-\Lambda \eta_{2}\right\|_{X} \leq \frac{\alpha}{m}\left\|y_{1}-y_{2}\right\|_{Y}+\frac{\beta}{m}\left\|\eta_{1}-\eta_{2}\right\|_{X} .
$$

Now using the assumptions on $R$ and $q$ we have

$$
\left\|y_{1}-y_{2}\right\|_{Y}=\left\|\mathcal{S}_{n}^{k} u^{k \eta_{1}}-\mathcal{S}_{n}^{k} u^{k \eta_{2}}\right\|_{Y} \leq c_{1} k\left\|\eta_{1}-\eta_{2}\right\|_{X},
$$

where the constant $c_{1}$ is given by (3.5). Thus

$$
\left\|\Lambda \eta_{1}-\Lambda \eta_{2}\right\|_{X} \leq\left(\frac{\alpha c_{1} k}{m}+\frac{\beta}{m}\right)\left\|\eta_{1}-\eta_{2}\right\|_{X} .
$$

Since $\beta<m$, by Banach fixed point theorem, $\Lambda$ has a unique fixed point $\eta^{*} \in K$ provided $k$ satisfies (3.6).

In the final step, we show the existence and uniqueness of the solution $u_{n}^{k}$ of (3.4). Let $\eta^{*} \in K$ be the fixed point of the operator $\Lambda$. It follows from (3.7) and (3.9) that

$$
y_{\eta^{*}}=\mathcal{S}_{n}^{k} u^{k \eta^{*}}, \quad u_{\eta^{*}}=\eta^{*} .
$$

Now, we write the inequality (3.8) for $\eta=\eta^{*}$, and use (3.14) to conclude that the function $u_{n}^{k}=\eta^{*} \in K$ is a solution of (3.4). The uniqueness of $u_{n}^{k}$ follows from the uniqueness of the fixed point of the operator $\Lambda$. 
For an error analysis, we have from (3.1) at $t=t_{n}$ that

$$
\left(A u_{n}, v-u_{n}\right)_{X}+\varphi\left(\mathcal{S}_{n} u, v\right)-\varphi\left(\mathcal{S}_{n} u, u_{n}\right)+j\left(u_{n}, v\right)-j\left(u_{n}, u_{n}\right) \geq\left(f_{n}, v-u_{n}\right)_{X} \quad \forall v \in K,
$$

where we have used the notation $\mathcal{S}_{n} u=\mathcal{S} u\left(t_{n}\right)$. Denote the error

$$
e_{n}^{k}:=u_{n}-u_{n}^{k}
$$

We take $v=u_{n}^{k}$ in (3.15), $v=u_{n}$ in (3.4), and add the two inequalities. After some manipulations, we obtain

$$
\begin{aligned}
m\left\|e_{n}^{k}\right\|_{X}^{2} \leq & \left(A u_{n}-A u_{n}^{k}, e_{n}^{k}\right) \\
\leq & \varphi\left(\mathcal{S}_{n}^{k} u^{k}, u_{n}\right)-\varphi\left(\mathcal{S}_{n}^{k} u^{k}, u_{n}^{k}\right)+\varphi\left(\mathcal{S}_{n} u, u_{n}^{k}\right)-\varphi\left(\mathcal{S}_{n} u, u_{n}\right) \\
& +j\left(u_{n}^{k}, u_{n}\right)-j\left(u_{n}^{k}, u_{n}^{k}\right)+j\left(u_{n}, u_{n}^{k}\right)-j\left(u_{n}, u_{n}\right) .
\end{aligned}
$$

From the assumptions made on $\varphi$ and $j$,

$$
\begin{gathered}
\varphi\left(\mathcal{S}_{n}^{k} u^{k}, u_{n}\right)-\varphi\left(\mathcal{S}_{n}^{k} u^{k}, u_{n}^{k}\right)+\varphi\left(\mathcal{S}_{n} u, u_{n}^{k}\right)-\varphi\left(\mathcal{S}_{n} u, u_{n}\right) \\
\leq \alpha\left(\left\|\mathcal{S}_{n}^{k} u^{k}-\mathcal{S}_{n}^{k} u\right\|_{Y}+\left\|\mathcal{S}_{n} u-\mathcal{S}_{n}^{k} u\right\|_{Y}\right)\left\|e_{n}^{k}\right\|_{X}, \\
j\left(u_{n}^{k}, u_{n}\right)-j\left(u_{n}^{k}, u_{n}^{k}\right)+j\left(u_{n}, u_{n}^{k}\right)-j\left(u_{n}, u_{n}\right) \leq \beta\left\|e_{n}^{k}\right\|_{X}^{2} .
\end{gathered}
$$

By the assumptions on $R(t)$ and $q$, we have

$$
\left\|\mathcal{S}_{n}^{k} u-\mathcal{S}_{n}^{k} v\right\|_{Y} \leq c_{1} k \sum_{j=0}^{n}\left\|u_{j}-v_{j}\right\|_{X}
$$

where the constant $c_{1}$ is independent of $k$ and $N$, and is given by (3.5). Now using (3.17)-(3.19) in (3.16), we get

$$
(m-\beta)\left\|e_{n}^{k}\right\|_{X} \leq \alpha c_{1} k \sum_{j=0}^{n}\left\|e_{j}^{k}\right\|_{X}+\alpha\left\|\mathcal{S}_{n} u-\mathcal{S}_{n}^{k} u\right\|_{Y} .
$$

In the rest of the paper, we use $c$ for a generic positive constant whose value may change from one occurrence to another, but is independent of discretization parameters $k$ and $h, h$ being a parameter for spatial discretization to be introduced in the next section.

Recall the following result (see [31], Lem. 2.32): Assume $\left\{g_{n}\right\}_{n=0}^{N}$ and $\left\{e_{n}\right\}_{n=0}^{N}$ are two sequences of nonnegative numbers satisfying

$$
e_{n} \leq \tilde{c} g_{n}+\bar{c} \sum_{j=0}^{n} k e_{j}, \quad n=0,1, \ldots, N .
$$

If $k$ is such that $\mathrm{e}^{-2 \bar{c} k} \leq 1-\bar{c} k$, then there exists a constant $c$ independent of $k$ and $N$ such that

$$
\max _{0 \leq n \leq N} e_{n} \leq c \max _{0 \leq n \leq N} g_{n}
$$

Since $m>\beta$, applying the above result on (3.20), we conclude that

$$
\max _{0 \leq n \leq N}\left\|\mathrm{e}_{n}^{k}\right\|_{X} \leq c \max _{0 \leq n \leq N}\left\|\mathcal{S}_{n} u-\mathcal{S}_{n}^{k} u\right\|_{Y}
$$

for all $k$ satisfying

$$
\mathrm{e}^{-2 c_{2} k} \leq 1-c_{2} k
$$

where $c_{2}=\alpha c_{1} /(m-\beta)$. 
Finally, assuming

$$
q \in C^{2}\left(\mathbb{R}_{+} \times \mathbb{R}_{+} ; \mathcal{L}(X, X)\right), \quad u \in W_{\text {loc }}^{2, \infty}\left(\mathbb{R}_{+} ; X\right),
$$

we have

$$
\begin{aligned}
\left\|\mathcal{S}_{n} u-\mathcal{S}_{n}^{k} u\right\|_{Y} & \leq c\left\|\int_{0}^{t_{n}} q\left(t_{n}, s\right) u(s) \mathrm{d} s-k \sum_{j=0}^{n} q\left(t_{n}, t_{j}\right) u_{j}\right\|_{X} \\
& \leq c k^{2}\left\|\left(\frac{\mathrm{d}}{\mathrm{d} s}\right)^{2}\left[q\left(t_{n}, s\right) u(s)\right]\right\|_{L^{\infty}((0, T) ; X)}
\end{aligned}
$$

and hence,

$$
\left\|\mathcal{S}_{n} u-\mathcal{S}_{n}^{k} u\right\|_{Y} \leq c k^{2}\|u\|_{W^{2, \infty}((0, T) ; X)} .
$$

To conclude, we have proved the following result.

Theorem 3.2. Assume that (2.2)-(2.6) and (2.8)-(2.10) hold and, moreover, assume the solution regularity (3.23). Then for the error of the semi-discrete solution of Problem 3.4, we have the estimate

$$
\max _{0 \leq n \leq N}\left\|u_{n}-u_{n}^{k}\right\|_{X} \leq c k^{2}
$$

provided that $k$ satisfies (3.6) and (3.22).

Recall that we have used the trapezoidal rule (3.2) for the discretization of the time integration. It follows from (3.25) that the resulting semi-discrete approximation has an $O\left(k^{2}\right)$ convergence rate provided $k$ is sufficiently small. Instead, if we use the approximation

$$
\int_{0}^{t_{n}} v(s) \mathrm{d} s \approx k \sum_{j=0}^{n-1} v_{j},
$$

then it can be proved that the corresponding semi-discrete scheme has a unique solution for all values of $k$. Using the arguments presented above, we also obtain an $O(k)$ error estimate for the numerical scheme that holds for any value of $k$.

\section{FULly Discrete APPROXIMATION}

In the fully discrete approximation, we discretize both the temporal and spatial variables. For the temporal discretization, we keep the notation and the assumptions used in Section 3. For the spatial discretization, we assume a regular family of finite element partitions of the spatial domain of the problem, and for each finite element partition $\left\{\mathcal{T}^{h}\right\}$, we introduce a finite element space $X^{h} \subseteq X$. Let $K^{h} \subseteq X^{h}$ be a non-empty, convex, and closed set to approximate $K$. In the following, we will concentrate on the case of internal approximation where

$$
K^{h} \subset K .
$$

For the application problems to be considered in Section 5, the above assumption does not impose serious restriction.

Then the fully discrete scheme for the problem (3.1) is to find the discrete solution $u^{k h}:=\left\{u_{n}^{k h}\right\}_{n=0}^{N} \subset K^{h}$ such that

$$
\begin{aligned}
& \left(A u_{n}^{k h}, v^{h}-u_{n}^{k h}\right)_{X}+\varphi\left(\mathcal{S}_{n}^{k h} u^{k h}, v^{h}\right)-\varphi\left(\mathcal{S}_{n}^{k h} u^{k h}, u_{n}^{k h}\right) \\
& \quad+j\left(u_{n}^{k h}, v^{h}\right)-j\left(u_{n}^{k h}, u_{n}^{k h}\right) \geq\left(f_{n}, v^{h}-u_{n}^{k h}\right)_{X} \quad \forall v^{h} \in K^{h},
\end{aligned}
$$


where

$$
\mathcal{S}_{n}^{k h} u^{k h}=R\left(t_{n}\right)\left(k \sum_{j=0}^{n} q\left(t_{n}, t_{j}\right) u_{j}^{k h}+a^{h}\right)
$$

and $a^{h} \in X^{h}$ is a finite element approximation of $a$.

Using the arguments presented in Theorem 3.1, we can show that under the conditions (2.2)-(2.6) and (2.8)(2.10), the problem (4.2) has a unique solution provided $k$ satisfies (3.6).

For an error analysis, we let $v=u_{n}^{k h}$ in (3.15) to obtain

$$
\begin{aligned}
& \left(A u_{n}, u_{n}^{k h}-u_{n}\right)_{X}+\varphi\left(\mathcal{S}_{n} u, u_{n}^{k h}\right)-\varphi\left(\mathcal{S}_{n} u, u_{n}\right) \\
& \quad+j\left(u_{n}, u_{n}^{k h}\right)-j\left(u_{n}, u_{n}\right) \geq\left(f_{n}, u_{n}^{k h}-u_{n}\right)_{X} .
\end{aligned}
$$

Denote the error

$$
e_{n}^{k h}:=u_{n}-u_{n}^{k h} .
$$

We then add (4.2) and (4.4). After some manipulations, we have

$$
\begin{aligned}
\left(A u_{n}-A u_{n}^{k h}, u_{n}-u_{n}^{k h}\right) \leq & R_{n}\left(v^{h}, u_{n}\right)+\left(A u_{n}-A u_{n}^{k h}, u_{n}-v^{h}\right) \\
& +\varphi\left(\mathcal{S}_{n} u, u_{n}^{k h}\right)-\varphi\left(\mathcal{S}_{n} u, u_{n}\right)+\varphi\left(\mathcal{S}_{n}^{k h} u^{k h}, u_{n}\right)-\varphi\left(\mathcal{S}_{n}^{k k} u^{k h}, u_{n}^{k h}\right) \\
& +\varphi\left(\mathcal{S}_{n}^{k h} u^{k h}, v^{h}\right)-\varphi\left(\mathcal{S}_{n}^{k h} u^{k h}, u_{n}\right)+\varphi\left(\mathcal{S}_{n} u, u_{n}\right)-\varphi\left(\mathcal{S}_{n} u, v^{h}\right) \\
& +j\left(u_{n}, u_{n}^{k h}\right)-j\left(u_{n}, u_{n}\right)+j\left(u_{n}^{k h}, u_{n}\right)-j\left(u_{n}^{k h}, u_{n}^{k h}\right) \\
& +j\left(u_{n}^{k h}, v^{h}\right)-j\left(u_{n}^{k h}, u_{n}\right)+j\left(u_{n}, u_{n}\right)-j\left(u_{n}, v^{h}\right),
\end{aligned}
$$

where

$$
\begin{aligned}
R_{n}\left(v^{h}, u\right):= & \left(A u_{n}, v^{h}-u_{n}\right)_{X}+\varphi\left(\mathcal{S}_{n} u, v^{h}\right)-\varphi\left(\mathcal{S}_{n} u, u_{n}\right) \\
& +j\left(u_{n}, v^{h}\right)-j\left(u_{n}, u_{n}\right)-\left(f_{n}, v^{h}-u_{n}\right)_{X} .
\end{aligned}
$$

Then

$$
\begin{aligned}
m\left\|e_{n}^{k h}\right\|_{X}^{2} \leq & R_{n}\left(v^{h}, u\right)+L\left\|e_{n}^{k h}\right\|_{X}\left\|u_{n}-v^{h}\right\|_{X} \\
& +\alpha\left\|\mathcal{S}_{n} u-\mathcal{S}_{n}^{k h} u^{k h}\right\|_{Y}\left(\left\|e_{n}^{k h}\right\|_{X}+\left\|u_{n}-v^{h}\right\|_{X}\right) \\
& +\beta\left\|e_{n}^{k h}\right\|_{X}^{2}+\beta\left\|e_{n}^{k h}\right\|_{X}\left\|u_{n}-v^{h}\right\|_{X} .
\end{aligned}
$$

Again, because of the assumption $m>\beta$, we can derive from the above inequality that

$$
\left\|e_{n}^{k h}\right\|_{X} \leq c\left[\left|R_{n}\left(v^{h}, u\right)\right|^{1 / 2}+\left\|u_{n}-v^{h}\right\|_{X}\right]+c_{3}\left\|\mathcal{S}_{n} u-\mathcal{S}_{n}^{k h} u^{k h}\right\|_{Y} \quad \forall v^{h} \in K^{h},
$$

where $c_{3}=\alpha /(m-\beta)+\sqrt{\alpha /(m-\beta)}$. Now write

$$
\left\|\mathcal{S}_{n} u-\mathcal{S}_{n}^{k h} u^{k h}\right\|_{Y} \leq\left\|\mathcal{S}_{n} u-\mathcal{S}_{n}^{k} u\right\|_{Y}+\left\|\mathcal{S}_{n}^{k} u-\mathcal{S}_{n}^{k h} u^{k h}\right\|_{Y} .
$$


The term $\left\|\mathcal{S}_{n} u-\mathcal{S}_{n}^{k} u\right\|_{Y}$ is bounded by (3.24), whereas for the term $\left\|\mathcal{S}_{n}^{k} u-\mathcal{S}_{n}^{k h} u^{k h}\right\|_{Y}$ we have

$$
\left\|\mathcal{S}_{n}^{k} u-\mathcal{S}_{n}^{k h} u^{k h}\right\|_{Y} \leq c_{1}\left(k \sum_{j=0}^{n}\left\|u_{j}-u_{j}^{k h}\right\|_{X}+\left\|a-a^{h}\right\|_{X}\right),
$$

where the constant $c_{1}$ is given by (3.5). Then from (4.6),

$$
\begin{aligned}
\left\|e_{n}^{k h}\right\|_{X} \leq & c\left[\left|R_{n}\left(v^{h}, u\right)\right|^{1 / 2}+\left\|u_{n}-v^{h}\right\|_{X}+\left\|a-a^{h}\right\|_{X}+k^{2}\right] \\
& +c_{4} k \sum_{j=0}^{n}\left\|e_{j}^{k h}\right\|_{X},
\end{aligned}
$$

where $c_{4}=c_{1} c_{3}$. Applying [31], Lemma 2.32 again we have

$$
\max _{0 \leq n \leq N}\left\|e_{n}^{k h}\right\|_{X} \leq c \max _{0 \leq n \leq N}\left[\left|R_{n}\left(v^{h}, u\right)\right|^{1 / 2}+\left\|u_{n}-v^{h}\right\|_{X}\right]+c\left\|a-a^{h}\right\|_{X}+c k^{2} \quad \forall v^{h} \in K^{h},
$$

provided $k$ satisfies

$$
\mathrm{e}^{-2 c_{4} k} \leq 1-c_{4} k
$$

To conclude, we have proved the following error estimate.

Theorem 4.1. Assume that (2.2)-(2.6) and (2.8)-(2.10) hold and, moreover, assume the solution regularity (3.23). Then the following error bound holds for the fully discrete solution of problem (4.2):

$$
\begin{aligned}
\max _{0 \leq n \leq N}\left\|u_{n}-u_{n}^{k h}\right\|_{X} \leq & c \max _{0 \leq n \leq N} \inf _{v^{h} \in K^{h}}\left[\left|R_{n}\left(v^{h}, u\right)\right|^{1 / 2}+\left\|u_{n}-v^{h}\right\|_{X}\right] \\
& +c\left\|a-a^{h}\right\|_{X}+c k^{2},
\end{aligned}
$$

for all $k$ satisfying (3.6) and (4.9).

Finally, we comment that if we use the discretization (3.26) for time integration, then the resulting fully discrete numerical approximation has a unique solution for all values of $k$. Using the arguments presented above, we also obtain the following error estimate for the numerical solution:

$$
\max _{0 \leq n \leq N}\left\|u_{n}-u_{n}^{k h}\right\|_{X} \leq c \max _{0 \leq n \leq N} \inf _{v^{h} \in K^{h}}\left[\left|R_{n}\left(v^{h}, u\right)\right|^{1 / 2}+\left\|u_{n}-v^{h}\right\|_{X}\right]+c\left\|a-a^{h}\right\|_{X}+c k,
$$

holding for any value of $k$.

\section{A frictional CONTACt PROBlem}

A large number of quasistatic contact problems with elastic and viscoelastic materials lead to a variational inequality of the form (2.1) in which the unknown is either the velocity or the displacement field. In both cases the abstract results in Sections 2-4 apply. In this section we illustrate the use of these results in the study of a contact problem in which the main variable is the velocity field.

The physical setting is the following. A viscoelastic body occupies a regular domain $\Omega$ of $\mathbb{R}^{d}(d=2,3)$ with surface $\Gamma$ that is partitioned into three disjoint measurable parts $\Gamma_{1}, \Gamma_{2}$ and $\Gamma_{3}$, such that meas $\left(\Gamma_{1}\right)>0$. We are interested in the evolution process of the mechanical state of the body in the unbounded interval of time $\mathbb{R}_{+}=[0,+\infty)$. The body is clamped on $\Gamma_{1}$ and so the displacement field vanishes there. Surface tractions of density $\boldsymbol{f}_{2}$ act on $\Gamma_{2}$ and volume forces of density $\boldsymbol{f}_{0}$ act in $\Omega$. We assume that the forces and tractions change 
slowly in time so that the acceleration of the system is negligible and, therefore, the process is quasistatic. Moreover, the body is in contact with an obstacle on $\Gamma_{3}$, the so called foundation. We assume that there is no separation between the body and the foundation, i.e. the contact is bilateral. This assumption is made only for definiteness, and note that our results above could be easily used in the study of various models which involve unilateral contact condition. The contact is frictional and is modeled with a slip-rate friction law.

We use the notation $\boldsymbol{x}=\left(x_{i}\right)$ for a generic point in $\Omega$ and we denote by $\boldsymbol{\nu}=\left(\nu_{i}\right)$ the unit outward normal vector on $\Gamma$. Here and below the indices $i, j, k, l$ run between 1 and $d$ and, unless stated otherwise, the summation convention over repeated indices is used. An index that follows a coma indicates a partial derivative with respect to the corresponding component of the spatial variable $\boldsymbol{x}$. We denote by $\boldsymbol{u}=\left(u_{i}\right), \boldsymbol{\sigma}=\left(\sigma_{i j}\right)$, and $\boldsymbol{\varepsilon}(\boldsymbol{u})=\left(\varepsilon_{i j}(\boldsymbol{u})\right)$ the displacement vector, the stress tensor, and the linearized strain tensor, respectively. Sometimes we do not indicate explicitly the dependence of functions on the spatial variable $\boldsymbol{x}$. Recall that components of the linearized strain tensor $\varepsilon(\boldsymbol{u})$ are given by

$$
\varepsilon_{i j}(\boldsymbol{u})=\frac{1}{2}\left(u_{i, j}+u_{j, i}\right),
$$

where $u_{i, j}=\partial u_{i} / \partial x_{j}$. We denote by $\mathbb{S}^{d}$ for the space of second order symmetric tensors on $\mathbb{R}^{d}$ or, equivalently, the space of symmetric matrices of order $d$. The canonical inner products and the corresponding norms on $\mathbb{R}^{d}$ and $\mathbb{S}^{d}$ are given by

$$
\begin{aligned}
& \boldsymbol{u} \cdot \boldsymbol{v}=u_{i} v_{i}, \quad\|\boldsymbol{v}\|=(\boldsymbol{v} \cdot \boldsymbol{v})^{1 / 2} \quad \forall \boldsymbol{u}=\left(u_{i}\right), \boldsymbol{v}=\left(v_{i}\right) \in \mathbb{R}^{d}, \\
& \boldsymbol{\sigma} \cdot \boldsymbol{\tau}=\sigma_{i j} \tau_{i j}, \quad\|\boldsymbol{\tau}\|=(\boldsymbol{\tau} \cdot \boldsymbol{\tau})^{1 / 2} \quad \forall \boldsymbol{\sigma}=\left(\sigma_{i j}\right), \boldsymbol{\tau}=\left(\tau_{i j}\right) \in \mathbb{S}^{d},
\end{aligned}
$$

respectively.

With these preliminaries, the classical formulation of the contact problem we consider in this section is the following.

Problem 5.1. Find a displacement $\boldsymbol{u}: \Omega \times \mathbb{R}_{+} \rightarrow \mathbb{R}^{d}$ and a stress field $\boldsymbol{\sigma}: \Omega \times \mathbb{R}_{+} \rightarrow \mathbb{S}^{d}$ such that, for all $t>0$,

$$
\begin{aligned}
& \begin{array}{ll}
\boldsymbol{\sigma}(t)=\mathcal{A} \varepsilon(\dot{\boldsymbol{u}}(t))+\mathcal{B} \varepsilon(\boldsymbol{u}(t)) & \text { in } \Omega,
\end{array} \\
& \begin{array}{ll}
\operatorname{Div} \boldsymbol{\sigma}(t)+\boldsymbol{f}_{0}(t)=\mathbf{0} & \text { in } \Omega,
\end{array} \\
& \begin{array}{ll}
\boldsymbol{u}(t)=\mathbf{0} & \text { on } \Gamma_{1},
\end{array} \\
& \begin{array}{ll}
\boldsymbol{\sigma}(t) \boldsymbol{\nu}=\boldsymbol{f}_{2}(t) & \text { on } \Gamma_{2},
\end{array} \\
& \begin{array}{ll}
u_{\nu}(t)=0 & \text { on } \Gamma_{3},
\end{array} \\
& \left.\begin{array}{ll}
\left\|\boldsymbol{\sigma}_{\tau}(t)\right\| \leq g\left(\left\|\dot{\boldsymbol{u}}_{\tau}(t)\right\|\right), & \\
-\boldsymbol{\sigma}_{\tau}(t)=g\left(\left\|\dot{\boldsymbol{u}}_{\tau}(t)\right\|\right) \frac{\dot{\boldsymbol{u}}_{\tau}(t)}{\left\|\boldsymbol{u}_{\tau}(t)\right\|} \quad \text { if } \quad \dot{\boldsymbol{u}}_{\tau}(t) \neq \mathbf{0}
\end{array}\right\} \quad \text { on } \Gamma_{3}, \\
& \begin{array}{ll}
\boldsymbol{u}(0)=\boldsymbol{u}_{0} & \text { in } \Omega .
\end{array}
\end{aligned}
$$

We present below a short description of the equations and conditions in Problem 5.1 and we refer the reader to $[14,29]$ for more details and mechanical interpretation. First, equation (5.1) represents the viscoelastic constitutive law in which $\mathcal{A}$ and $\mathcal{B}$ are given nonlinear operators, called the viscosity operator and elasticity operator, respectively. Equality (5.2) represents the equilibrium equation where Div is the divergence operator, i.e. Div $\boldsymbol{\sigma}=\left(\sigma_{i j, j}\right)$. Conditions (5.3) and (5.4) are the displacement and traction boundary conditions, respectively. Condition (5.5) represents the contact condition in which $u_{\nu}$ denotes the normal component of the displacement field. We use it here since the contact is assumed to be bilateral. Condition (5.6) represents a version of Coulomb's law of dry friction, in which $\boldsymbol{\sigma}_{\tau}$ is the tangential traction, $\dot{\boldsymbol{u}}_{\tau}$ is the tangential part of 
the velocity field, the so-called slip-rate, and $g$ is the friction bound, assumed to depend on the norm of the slip-rate. Considering such kind of slip-rate dependent friction law represents the novelty of the mathematical model (5.1)-(5.7). Finally, (5.7) represents the initial condition in which the function $\boldsymbol{u}_{0}$ denotes the initial displacement field.

In the study of the contact problem (5.1)-(5.7) we use standard notation for Lebesgue's spaces and Sobolev's spaces associated to $\Omega$ and $\Gamma$. For all $\boldsymbol{v} \in H^{1}(\Omega)^{d}$ we still denote by $\boldsymbol{v}$ the trace of $\boldsymbol{v}$ on $\Gamma$ and we use the notation $v_{\nu}$ and $\boldsymbol{v}_{\tau}$ for the normal and tangential components of $\boldsymbol{v}$ on $\Gamma$ given by

$$
v_{\nu}=\boldsymbol{v} \cdot \boldsymbol{\nu}, \quad \boldsymbol{v}_{\tau}=\boldsymbol{v}-v_{\nu} \boldsymbol{\nu} .
$$

We recall that the normal and tangential components of the stress field $\boldsymbol{\sigma}$ on the boundary are defined by

$$
\sigma_{\nu}=(\sigma \nu) \cdot \nu, \quad \sigma_{\tau}=\sigma \nu-\sigma_{\nu} \nu
$$

If $\boldsymbol{\sigma}$ is a regular function, then the following Green's formula holds:

$$
\int_{\Omega} \boldsymbol{\sigma} \cdot \boldsymbol{\varepsilon}(\boldsymbol{v}) \mathrm{d} x+\int_{\Omega} \operatorname{Div} \boldsymbol{\sigma} \cdot \boldsymbol{v} \mathrm{d} x=\int_{\Gamma} \boldsymbol{\sigma} \boldsymbol{\nu} \cdot \boldsymbol{v} \mathrm{d} a \quad \forall \boldsymbol{v} \in H^{1}(\Omega)^{d} .
$$

Next, we introduce the spaces $V$ and $Q$, defined by

$$
\begin{aligned}
& V=\left\{\boldsymbol{v}=\left(v_{i}\right) \in H^{1}(\Omega)^{d}: \boldsymbol{v}=\mathbf{0} \text { a.e. on } \Gamma_{1}, v_{\nu}=0 \text { a.e. on } \Gamma_{3}\right\}, \\
& Q=\left\{\boldsymbol{\tau}=\left(\tau_{i j}\right) \in L^{2}(\Omega)^{d \times d}: \tau_{i j}=\tau_{j i}, 1 \leq i, j \leq d\right\} .
\end{aligned}
$$

The space $Q$ is a real Hilbert space with the canonical inner product

$$
(\boldsymbol{\sigma}, \boldsymbol{\tau})_{Q}=\int_{\Omega} \sigma_{i j}(\boldsymbol{x}) \tau_{i j}(\boldsymbol{x}) \mathrm{d} x .
$$

The associated norm is denoted by $\|\cdot\|_{Q}$. Since meas $\left(\Gamma_{1}\right)>0$, it is well known that $V$ is a real Hilbert space with the inner product

$$
(\boldsymbol{u}, \boldsymbol{v})_{V}=(\varepsilon(\boldsymbol{u}), \boldsymbol{\varepsilon}(\boldsymbol{v}))_{Q} \quad \forall \boldsymbol{u}, \boldsymbol{v} \in V .
$$

The corresponding norm is $\|\cdot\|_{V}$. By the Sobolev trace theorem, there exists a positive constant $c_{0}$, depending on $\Omega, \Gamma_{1}$, and $\Gamma_{3}$, such that

$$
\|\boldsymbol{v}\|_{L^{2}\left(\Gamma_{3}\right)^{d}} \leq c_{0}\|\boldsymbol{v}\|_{V} \quad \forall \boldsymbol{v} \in V .
$$

We list now the assumptions on the data. Assume the viscosity operator $\mathcal{A}$ and the elasticity operator $\mathcal{B}$ satisfy

( (a) $\mathcal{A}: \Omega \times \mathbb{S}^{d} \rightarrow \mathbb{S}^{d}$.

(b) There exists $L_{\mathcal{A}}>0$ such that

$$
\begin{aligned}
&\left\|\mathcal{A}\left(\boldsymbol{x}, \varepsilon_{1}\right)-\mathcal{A}\left(\boldsymbol{x}, \varepsilon_{2}\right)\right\| \leq L_{\mathcal{A}}\left\|\varepsilon_{1}-\varepsilon_{2}\right\| \\
& \forall \varepsilon_{1}, \varepsilon_{2} \in \mathbb{S}^{d}, \text { a.e. } \boldsymbol{x} \in \Omega .
\end{aligned}
$$

(c) There exists $m_{\mathcal{A}}>0$ such that

$$
\begin{aligned}
& \left(\mathcal{A}\left(\boldsymbol{x}, \varepsilon_{1}\right)-\mathcal{A}\left(\boldsymbol{x}, \varepsilon_{2}\right)\right) \cdot\left(\varepsilon_{1}-\varepsilon_{2}\right) \geq m_{\mathcal{A}}\left\|\varepsilon_{1}-\varepsilon_{2}\right\|^{2} \\
& \forall \varepsilon_{1}, \varepsilon_{2} \in \mathbb{S}^{d}, \text { a.e. } \boldsymbol{x} \in \Omega .
\end{aligned}
$$

(d) The mapping $\boldsymbol{x} \mapsto \mathcal{A}(\boldsymbol{x}, \boldsymbol{\varepsilon})$ is measurable on $\Omega$, for any $\varepsilon \in \mathbb{S}^{d}$.

(e) The mapping $\boldsymbol{x} \mapsto \mathcal{A}(\boldsymbol{x}, \mathbf{0})$ belongs to $Q$. 


$$
\left\{\begin{array}{l}
\text { (a) } \mathcal{B}: \Omega \times \mathbb{S}^{d} \rightarrow \mathbb{S}^{d} . \\
\text { (b) There exists } L_{\mathcal{B}}>0 \text { such that } \\
\left\|\mathcal{B}\left(\boldsymbol{x}, \varepsilon_{1}\right)-\mathcal{B}\left(\boldsymbol{x}, \varepsilon_{2}\right)\right\| \leq L_{\mathcal{B}}\left\|\varepsilon_{1}-\varepsilon_{2}\right\| \\
\forall \varepsilon_{1}, \varepsilon_{2} \in \mathbb{S}^{d}, \text { a.e. } \boldsymbol{x} \in \Omega . \\
\text { (c) The mapping } \boldsymbol{x} \mapsto \mathcal{B}(\boldsymbol{x}, \boldsymbol{\varepsilon}) \text { is measurable on } \Omega, \\
\text { for any } \boldsymbol{\varepsilon} \in \mathbb{S}^{d} . \\
\text { (d) The mapping } \boldsymbol{x} \mapsto \mathcal{B}(\boldsymbol{x}, \boldsymbol{0}) \text { belongs to } Q .
\end{array}\right.
$$

We also assume that the forces and tractions satisfy

$$
\boldsymbol{f}_{0} \in C\left(\mathbb{R}_{+} ; L^{2}(\Omega)^{d}\right), \quad \boldsymbol{f}_{2} \in C\left(\mathbb{R}_{+} ; L^{2}\left(\Gamma_{2}\right)^{d}\right),
$$

and the friction bound verifies the condition

$$
\left\{\begin{array}{l}
\text { (a) } g: \Gamma_{3} \times \mathbb{R}_{+} \rightarrow \mathbb{R}_{+} . \\
\text {(b) There exists } L_{g}>0 \text { such that } \\
\left|g\left(\boldsymbol{x}, r_{1}\right)-g\left(\boldsymbol{x}, r_{2}\right)\right| \leq L_{g}\left|r_{1}-r_{2}\right| \\
\quad \forall r_{1}, r_{2} \in \mathbb{R}, \text { a.e. } \boldsymbol{x} \in \Omega . \\
\text { (c) The mapping } \boldsymbol{x} \mapsto g(\boldsymbol{x}, r) \text { is measurable on } \Gamma_{3}, \\
\quad \text { for any } r \in \mathbb{R} . \\
\text { (d) The mapping } \boldsymbol{x} \mapsto g(\boldsymbol{x}, 0) \text { belongs to } L^{2}\left(\Gamma_{3}\right) .
\end{array}\right.
$$

Finally, for the initial displacement, assume

$$
\boldsymbol{u}_{0} \in V \text {. }
$$

If $(\boldsymbol{u}, \boldsymbol{\sigma})$ is a sufficiently regular functions satisfying (5.1)-(5.7), then, using (5.8)-(5.10) we can deduce that for $t \in \mathbb{R}_{+}$,

$$
\begin{aligned}
& \int_{\Omega} \mathcal{A} \varepsilon(\dot{\boldsymbol{u}}(t)) \cdot(\varepsilon(\boldsymbol{v})-\varepsilon(\dot{\boldsymbol{u}}(t))) \mathrm{d} x+\int_{\Omega} \mathcal{B} \varepsilon(\boldsymbol{u}(t)) \cdot(\varepsilon(\boldsymbol{v})-\varepsilon(\dot{\boldsymbol{u}}(t))) \mathrm{d} x \\
& \quad+\int_{\Gamma_{3}} g\left(\left\|\dot{\boldsymbol{u}}_{\tau}(t)\right\|\right)\left\|\boldsymbol{v}_{\tau}\right\| \mathrm{d} a-\int_{\Gamma_{3}} g\left(\left\|\dot{\boldsymbol{u}}_{\tau}(t)\right\|\right)\left\|\dot{\boldsymbol{u}}_{\tau}(t)\right\| \mathrm{d} a \\
& \quad \geq \int_{\Omega} \boldsymbol{f}_{0}(t) \cdot(\boldsymbol{v}-\dot{\boldsymbol{u}}(t)) \mathrm{d} x+\int_{\Gamma_{2}} \boldsymbol{f}_{2}(t) \cdot(\boldsymbol{v}-\dot{\boldsymbol{u}}(t)) \mathrm{d} a \quad \forall \boldsymbol{v} \in V .
\end{aligned}
$$

Define an operator $A: V \rightarrow V$, functionals $\varphi: V \times V \rightarrow \mathbb{R}, j: V \times V \rightarrow \mathbb{R}$ and a function $f: \mathbb{R}_{+} \rightarrow V$ as follows:

$$
\begin{aligned}
& (A \boldsymbol{u}, \boldsymbol{v})_{V}=(\mathcal{A} \varepsilon(\boldsymbol{u}), \boldsymbol{\varepsilon}(\boldsymbol{v}))_{Q} \quad \forall \boldsymbol{u}, \boldsymbol{v} \in V, \\
& \varphi(\boldsymbol{u}, \boldsymbol{v})=(\mathcal{B} \varepsilon(\boldsymbol{u}), \varepsilon(\boldsymbol{v}))_{Q} \quad \forall \boldsymbol{u}, \boldsymbol{v} \in V, \\
& j(\boldsymbol{u}, \boldsymbol{v})=\int_{\Gamma_{3}} g\left(\left\|\boldsymbol{u}_{\tau}(t)\right\|\right)\left\|\boldsymbol{v}_{\tau}\right\| \mathrm{d} a \quad \forall \boldsymbol{u}, \boldsymbol{v} \in V, \\
& (\boldsymbol{f}(t), \boldsymbol{v})_{V}=\int_{\Omega} \boldsymbol{f}_{0}(t) \cdot \boldsymbol{v} \mathrm{d} x+\int_{\Gamma_{2}} \boldsymbol{f}_{2}(t) \cdot \boldsymbol{v} \mathrm{d} a \quad \forall \boldsymbol{v} \in V, t \in \mathbb{R}_{+} .
\end{aligned}
$$


Using the notation (5.19)-(5.22) we see that the inequality (5.18) can be written as

$$
\begin{aligned}
& (A \dot{\boldsymbol{u}}(t), \boldsymbol{v}-\dot{\boldsymbol{u}}(t))_{V}+\varphi(\boldsymbol{u}(t), \boldsymbol{v})-\varphi(\boldsymbol{u}(t), \dot{\boldsymbol{u}}(t))_{V} \\
& \quad+j(\dot{\boldsymbol{u}}(t), \boldsymbol{v})-j(\dot{\boldsymbol{u}}(t), \dot{\boldsymbol{u}}(t))_{V} \geq(\boldsymbol{f}(t), \boldsymbol{v}-\dot{\boldsymbol{u}}(t))_{V} \quad \forall \boldsymbol{v} \in V .
\end{aligned}
$$

Define an operator $\mathcal{S}: C\left(\mathbb{R}_{+} ; V\right) \rightarrow C\left(\mathbb{R}_{+} ; V\right)$ by

$$
\mathcal{S} \boldsymbol{v}(t)=\int_{0}^{t} \boldsymbol{v}(s) \mathrm{d} s+\boldsymbol{u}_{0} \quad \forall \boldsymbol{v} \in C\left(\mathbb{R}_{+}, V\right), t \in \mathbb{R}_{+}
$$

and let $\boldsymbol{w}=\dot{\boldsymbol{u}}$ denote the velocity field. Then, the initial condition $\boldsymbol{u}(0)=\boldsymbol{u}_{0}$ and (5.24) imply that

$$
\boldsymbol{u}(t)=\mathcal{S} \boldsymbol{w}(t) \quad \forall t \in \mathbb{R}_{+} .
$$

Therefore, (5.23) leads to the following variational formulation of problem (5.1)-(5.7), in terms of velocity.

Problem 5.2. Find a velocity field $\boldsymbol{w}: \mathbb{R}_{+} \rightarrow V$ such that, for all $t \in \mathbb{R}_{+}$,

$$
\begin{aligned}
& (A \boldsymbol{w}(t), \boldsymbol{v}-\boldsymbol{w}(t))_{V}+\varphi(\mathcal{S} \boldsymbol{w}(t), \boldsymbol{v})-\varphi(\mathcal{S} \boldsymbol{w}(t), \boldsymbol{w}(t)) \\
& \quad+j(\boldsymbol{w}(t), \boldsymbol{v})-j(\boldsymbol{w}(t), \boldsymbol{w}(t)) \geq(\boldsymbol{f}(t), \boldsymbol{v}-\boldsymbol{w}(t))_{V} \quad \forall \boldsymbol{v} \in V .
\end{aligned}
$$

In the study of Problem 5.2 we have the following result.

Theorem 5.3. Assume (5.13)-(5.17). Then, there exists $L_{0}>0$ depending only on $\Omega, \Gamma_{1}, \Gamma_{3}$ and $\mathcal{A}$ such that if $L_{g}<L_{0}$, Problem 5.2 has a unique solution $\boldsymbol{w} \in C\left(\mathbb{R}_{+} ; V\right)$. Moreover, if for some $p \in[1, \infty]$,

$$
\boldsymbol{f}_{0} \in W_{\mathrm{loc}}^{1, p}\left(\mathbb{R}_{+} ; L^{2}(\Omega)^{d}\right), \quad \boldsymbol{f}_{2} \in W_{\mathrm{loc}}^{1, p}\left(\mathbb{R}_{+} ; L^{2}\left(\Gamma_{2}\right)^{d}\right),
$$

then the solution satisfies $\boldsymbol{w} \in W_{\text {loc }}^{1, p}\left(\mathbb{R}_{+} ; V\right)$.

Proof. It is straightforward to see that inequality (5.26) represents a quasivariational inequality of the form (2.1) in which $X=Y=K=V$. Therefore, in order to apply Theorems 2.1 and 2.2, we note that by assumption (5.13) it follows that $A$ is a strongly monotone Lipschitz continuous operator, i.e. it satisfies conditions (2.3) with $m=m_{\mathcal{A}}$ and $L=L_{\mathcal{A}}$. Moreover, from (5.14) it follows that the function $\varphi$ defined by (5.20) satisfies (2.4)(a) and, in addition,

$$
\varphi\left(\boldsymbol{u}_{1}, \boldsymbol{v}_{2}\right)-\varphi\left(\boldsymbol{u}_{1}, \boldsymbol{v}_{1}\right)+\varphi\left(\boldsymbol{u}_{2}, \boldsymbol{v}_{1}\right)-\varphi\left(\boldsymbol{u}_{2}, \boldsymbol{v}_{2}\right) \leq L_{\mathcal{B}}\left\|\boldsymbol{u}_{1}-\boldsymbol{u}_{2}\right\|_{V}\left\|\boldsymbol{v}_{1}-\boldsymbol{v}_{2}\right\|_{V} \quad \forall \boldsymbol{u}_{1}, \boldsymbol{u}_{2}, \boldsymbol{v}_{1}, \boldsymbol{v}_{2} \in V,
$$

which shows that (2.4)(b) holds, too. Next, we use (5.16) and (5.12) to see that the function $j$ defined by (5.21) satisfies (2.5)(a) and, in addition,

$$
j\left(\boldsymbol{u}_{1}, \boldsymbol{v}_{2}\right)-j\left(\boldsymbol{u}_{1}, \boldsymbol{v}_{1}\right)+j\left(\boldsymbol{u}_{2}, \boldsymbol{v}_{1}\right)-j\left(\boldsymbol{u}_{2}, \boldsymbol{v}_{2}\right) \leq c_{0}^{2} L_{g}\left\|\boldsymbol{u}_{1}-\boldsymbol{u}_{2}\right\|_{V}\left\|\boldsymbol{v}_{1}-\boldsymbol{v}_{2}\right\|_{V} \quad \forall \boldsymbol{u}_{1}, \boldsymbol{u}_{2}, \boldsymbol{v}_{1}, \boldsymbol{v}_{2} \in V .
$$

This inequality shows that $j$ satisfies condition (2.5)(b) with $\beta=c_{0}^{2} L_{g}$. Finally, we note that the operator (5.24) verifies condition (2.7) and assumption (5.15) implies that $\boldsymbol{f} \in C\left(\mathbb{R}_{+} ; V\right)$ and, therefore, condition (2.8) holds.

Assume now that $c_{0}^{2} L_{g}<m_{\mathcal{A}}$ and note that in this case condition (2.6) is satisfied. Then, applying Theorem 2.1 we conclude that inequality (5.26) has a unique solution $\boldsymbol{w} \in C\left(\mathbb{R}_{+} ; V\right)$ and we may take $L_{0}=m_{\mathcal{A}} / c_{0}^{2}$. Recall that $c_{0}$ depends only on $\Omega, \Gamma_{1}$ and $\Gamma_{3}$ and, therefore, $L_{0}$ depends only on $\Omega, \Gamma_{1}, \Gamma_{3}$ and $\mathcal{A}$.

Finally, we note that assumption (5.27) implies that $f \in W_{\text {loc }}^{1, p}\left(\mathbb{R}_{+} ; V\right)$ and, in addition, the operator $\mathcal{S}$ defined by (5.24) satisfies the regularity assumption (2.13). Therefore, by Theorem 2.2 we deduce that if (5.27) holds then $\boldsymbol{w} \in W_{\text {loc }}^{1, p}\left(\mathbb{R}_{+} ; V\right)$, which completes the proof. 
Let $\boldsymbol{w}$ denote a solution of Problem 5.2 and define $\boldsymbol{u}$ and $\boldsymbol{\sigma}$ by (5.25), (5.24) and (5.1). Then, the couple $(\boldsymbol{u}, \boldsymbol{\sigma})$ is called a weak solution of the frictional contact problem (5.1)-(5.7). It follows from Theorem 5.3 that if (5.13)(5.17) hold and the Lipschitz constant $L_{g}$ is small enough, then the contact problem (5.1)-(5.7) has a unique weak solution. Moreover, using (5.13), (5.14) and (5.17) we see that the weak solution satisfies $\boldsymbol{u} \in C^{1}\left(\mathbb{R}_{+} ; V\right)$ and $\sigma \in C\left(\mathbb{R}_{+} ; Q\right)$. Theorem 5.3 also shows that, if in addition (5.27) holds, then the weak solution has the regularity $\boldsymbol{u} \in W_{\mathrm{loc}}^{2, p}\left(\mathbb{R}_{+} ; V\right)$ and $\boldsymbol{\sigma} \in W_{\mathrm{loc}}^{1, p}\left(\mathbb{R}_{+} ; Q\right)$.

\section{Numerical ANALYSis OF CONTACT PROBLEM}

We now apply the results from Sections 3 and 4 for numerical analysis of the contact problem of Section 5 . For computational purposes, the numerical solution will be considered on the domain $[0, T] \times \Omega$, where $T$ is an arbitrarily large constant. We assume that $\Omega$ is a polyhedral domain and the regular family $\left\{\mathcal{T}^{h}\right\}$ of triangular finite element partitions of $\bar{\Omega}$ are compatible with the boundary decomposition $\Gamma=\overline{\Gamma_{1}} \cup \overline{\Gamma_{2}} \cup \overline{\Gamma_{3}}$, i.e., if a side of an element $T \in \mathcal{T}^{h}$ lies on the boundary $\Gamma$, then the side lies entirely in $\overline{\Gamma_{1}}, \overline{\Gamma_{2}}$ or $\overline{\Gamma_{3}}$. Let $X^{h} \subseteq H^{1}(\Omega)^{d}$ be the finite element space of linear functions corresponding to the partition $\mathcal{T}^{h}$. The space $V$ is approximated by the following finite element space:

$$
V^{h}=\left\{\boldsymbol{v}^{h} \in X^{h}: \boldsymbol{v}^{h}=\mathbf{0} \text { at the nodes on } \overline{\Gamma_{1}}, v_{\nu}^{h}=0 \text { at the nodes on } \overline{\Gamma_{3}}\right\} .
$$

The fully discrete approximation of Problem 5.2 is to find the discrete velocity field $\boldsymbol{w}^{k h}:=\left\{\boldsymbol{w}_{n}^{k h}\right\}_{n=0}^{N} \subset V^{h}$ such that

$$
\begin{aligned}
& \left(A \boldsymbol{w}_{n}^{k h}, \boldsymbol{v}^{h}-\boldsymbol{w}_{n}^{k h}\right)_{V}+\varphi\left(\mathcal{S}_{n}^{k h} \boldsymbol{w}^{k h}, \boldsymbol{v}^{h}\right)-\varphi\left(\mathcal{S}_{n}^{k h} \boldsymbol{w}^{k h}, \boldsymbol{w}_{n}^{k h}\right) \\
& \quad+j\left(\boldsymbol{w}_{n}^{k h}, \boldsymbol{v}^{h}\right)-j\left(\boldsymbol{w}_{n}^{k h}, \boldsymbol{w}_{n}^{k h}\right) \geq\left(\boldsymbol{f}_{n}, \boldsymbol{v}^{h}-\boldsymbol{w}_{n}^{k h}\right)_{V} \quad \forall \boldsymbol{v}^{h} \in V^{h},
\end{aligned}
$$

where

$$
\mathcal{S}_{n}^{k h} \boldsymbol{w}^{k h}=k \sum_{j=0}^{n} \boldsymbol{w}_{j}^{k h}+\boldsymbol{u}_{0}^{h}
$$

and $\boldsymbol{u}_{0}^{h} \in V^{h}$ is a finite element approximation of $\boldsymbol{u}_{0}$.

Problem (6.1) has a unique solution under the conditions stated in Theorem 5.3 provided that $k$ satisfies (3.6). To derive error estimates, we make the following solution regularity assumptions:

$$
\begin{aligned}
\boldsymbol{\sigma} \boldsymbol{\nu} & \in C\left(\mathbb{R}_{+} ; L^{2}(\Gamma)^{d}\right), \\
\boldsymbol{w}_{\tau} & \in C\left(\mathbb{R}_{+} ; \tilde{H}^{2}\left(\Gamma_{3}\right)^{d}\right), \\
\boldsymbol{w} & \in W_{\mathrm{loc}}^{2, \infty}\left(\mathbb{R}_{+} ; V\right), \\
\boldsymbol{w} & \in C\left(\mathbb{R}_{+} ; H^{2}(\Omega)^{d}\right), \\
\boldsymbol{u}_{0} & \in H^{2}(\Omega)^{d} .
\end{aligned}
$$

Here the space $\tilde{H}^{2}\left(\Gamma_{3}\right)$ is defined as follows: Let $\Gamma_{3}$ be represented as $\Gamma_{3}=\cup_{1 \leq i \leq I} \Gamma_{3, i}$ with each $\Gamma_{3, i}$ a closed subset of an affine hyperplane in $\mathbb{R}^{d}$. Then $\tilde{H}^{2}\left(\Gamma_{3}\right)$ consists of functions $v$ such that $v \in H^{2}\left(\Gamma_{3, i}\right), 1 \leq i \leq I$. We let

$$
\|v\|_{\tilde{H}^{2}\left(\Gamma_{3}\right)}=\left(\sum_{i=1}^{I}\|v\|_{H^{2}\left(\Gamma_{3, i}\right)}^{2}\right)^{1 / 2} .
$$


Under the solution regularity (6.3), the following pointwise relations hold, for all $t \in \mathbb{R}_{+}$:

$$
\begin{aligned}
\operatorname{Div} \boldsymbol{\sigma}(t)+\boldsymbol{f}_{0}(t) & =\mathbf{0} \quad \text { a.e. in } \Omega, \\
\boldsymbol{\sigma}(t) \boldsymbol{\nu} & =\boldsymbol{f}_{2}(t) \quad \text { a.e. on } \Gamma_{2} .
\end{aligned}
$$

Moreover, from (4.10) we have the error estimate

$$
\max _{0 \leq n \leq N}\left\|\boldsymbol{w}_{n}-\boldsymbol{w}_{n}^{k h}\right\|_{V} \leq c \max _{0 \leq n \leq N} \inf _{\boldsymbol{v}^{h} \in V^{h}}\left[\left|R_{n}\left(\boldsymbol{v}^{h}, \boldsymbol{w}\right)\right|^{1 / 2}+\left\|\boldsymbol{w}_{n}-\boldsymbol{v}^{h}\right\|_{V}\right]+c\left\|\boldsymbol{u}_{0}-\boldsymbol{u}_{0}^{h}\right\|_{V}+c k^{2}
$$

where

$$
R_{n}\left(\boldsymbol{v}^{h}, \boldsymbol{w}\right)=\left(\boldsymbol{\sigma}_{n}, \boldsymbol{\varepsilon}\left(\boldsymbol{v}^{h}-\boldsymbol{w}_{n}\right)\right)_{Q}+j\left(\boldsymbol{w}_{n}, \boldsymbol{v}^{h}\right)-j\left(\boldsymbol{w}_{n}, \boldsymbol{w}_{n}\right)-\left(\boldsymbol{f}_{n}, \boldsymbol{v}^{h}-\boldsymbol{w}_{n}\right)_{V}
$$

Applying Green's formula (5.10) on the first term and then using the pointwise relations (6.8)-(6.9), we have

$$
R_{n}\left(\boldsymbol{v}^{h}, \boldsymbol{w}\right)=\int_{\Gamma_{3}} \boldsymbol{\sigma}_{n \tau} \cdot\left(\boldsymbol{v}_{\tau}^{h}-\boldsymbol{w}_{n \tau}\right) \mathrm{d} a+\int_{\Gamma_{3}} g\left(\left\|\boldsymbol{w}_{n \tau}\right\|\right)\left(\left\|\boldsymbol{v}_{\tau}^{h}\right\|-\left\|\boldsymbol{w}_{n \tau}\right\|\right) \mathrm{d} a,
$$

which implies that

$$
\left|R_{n}\left(\boldsymbol{v}^{h}, \boldsymbol{w}\right)\right| \leq\left(\left\|\boldsymbol{\sigma}_{n \tau}\right\|_{L^{2}\left(\Gamma_{3}\right)^{d}}+\left\|g\left(\left\|\boldsymbol{w}_{n \tau}\right\|\right)\right\|_{L^{2}\left(\Gamma_{3}\right)}\right)\left\|\boldsymbol{w}_{n \tau}-\boldsymbol{v}_{\tau}^{h}\right\|_{L^{2}\left(\Gamma_{3}\right)^{d}}
$$

Thus, from (6.10), we obtain

$$
\max _{0 \leq n \leq N}\left\|\boldsymbol{w}_{n}-\boldsymbol{w}_{n}^{k h}\right\|_{V} \leq c \max _{0 \leq n \leq N} \inf _{\boldsymbol{v}^{h} \in K^{h}}\left(\left\|\boldsymbol{w}_{n \tau}-\boldsymbol{v}_{\tau}^{h}\right\|_{L^{2}\left(\Gamma_{3}\right)^{d}}^{1 / 2}+\left\|\boldsymbol{w}_{n}-\boldsymbol{v}^{h}\right\|_{V}\right)+c\left\|\boldsymbol{u}_{0}-\boldsymbol{u}_{0}^{h}\right\|_{V}+c k^{2}
$$

Under the solution regularity assumptions (6.3)-(6.7), we can apply standard finite element interpolation error estimates (see e.g., $[5,6,8]$ ) to see that each of the terms

$$
\left\|\boldsymbol{u}_{0}-\boldsymbol{u}_{0}^{h}\right\|_{V} \quad \text { and } \max _{0 \leq n \leq N} \inf _{\boldsymbol{v}^{h} \in K^{h}}\left(\left\|\boldsymbol{w}_{n \tau}-\boldsymbol{v}_{\tau}^{h}\right\|_{L^{2}\left(\Gamma_{3}\right)^{d}}^{1 / 2}+\left\|\boldsymbol{w}_{n}-\boldsymbol{v}^{h}\right\|_{V}\right)
$$

is bounded by $h$ multiplied by a constant depending on certain norm of the solution. Hence, we get the following error bound for the fully discrete numerical solution of Problem 5.2:

$$
\max _{0 \leq n \leq N}\left\|\boldsymbol{w}_{n}-\boldsymbol{w}_{n}^{h k}\right\|_{V} \leq c\left(h+k^{2}\right)
$$

for all $k$ satisfying (3.6) and (4.9).

Finally, note that if $\boldsymbol{w}$ is the solution of Problem 5.2, then the displacement field $\boldsymbol{u}$ of the frictional contact problem (5.1)-(5.7) is

$$
\boldsymbol{u}(t)=\int_{0}^{t} \boldsymbol{w}(s) \mathrm{d} s+\boldsymbol{u}_{0} \quad \forall t \in \mathbb{R}_{+} .
$$

Its fully discrete approximation $\left\{\boldsymbol{u}_{n}^{k h}\right\}_{n \geq 0}$ is given by

$$
\boldsymbol{u}_{n}^{k h}=k \sum_{j=0}^{n} \boldsymbol{w}_{j}^{k h}+\boldsymbol{u}_{0}^{h}
$$

Then, by applying a standard technique (see e.g. [14], Chap. 7), we have

$$
\left\|\boldsymbol{u}_{n}-\boldsymbol{u}_{n}^{k h}\right\|_{V} \leq c k^{2}+\left\|\boldsymbol{u}_{0}-\boldsymbol{u}_{0}^{h}\right\|_{V}+c k \sum_{j=1}^{n}\left\|\boldsymbol{w}_{j}-\boldsymbol{w}_{j}^{k h}\right\|_{V}
$$


which implies that

$$
\max _{0 \leq n \leq N}\left\|\boldsymbol{u}_{n}-\boldsymbol{u}_{n}^{k h}\right\|_{V} \leq c k^{2}+\left\|\boldsymbol{u}_{0}-\boldsymbol{u}_{0}^{h}\right\|_{V}+c \max _{0 \leq n \leq N}\left\|\boldsymbol{w}_{n}-\boldsymbol{w}_{n}^{k h}\right\|_{V} .
$$

We conclude that under the solution regularity (6.3)-(6.7) we have an error bound of the size $O\left(h+k^{2}\right)$ for the displacement field $\boldsymbol{u}$ of the Problem 5.1.

\section{Numerical Simulations}

This section is devoted to numerical simulations of the frictional contact Problem 5.1 presented in Section 5 . First, we describe the numerical solution of Problem 5.1. Then we present some numerical examples in order to reconfirm the error estimates obtained in the previous section. And, finally, we provide some comments concerning the behavior of the solution of the frictional problem considered. Everywhere below we assume that the conditions stated in Theorem 5.3 hold and we use the notation introduced in Section 5.

Numerical solution. We start by providing an equivalent variational formulation of the Problem 5.1 well suited for numerical treatment. To this end we note that, for all $t \in \mathbb{R}_{+}$, conditions (5.6) are equivalent to the subdifferential inclusion

$$
-\boldsymbol{\sigma}_{\tau}(t) \in g\left(\left\|\dot{\boldsymbol{u}}_{\tau}(t)\right\|\right) \partial\left\|\dot{\boldsymbol{u}}_{\tau}(t)\right\| \quad \text { on } \quad \Gamma_{3},
$$

in which $\partial \varphi$ denotes the subdifferential of the convex function $\varphi$. Next, we consider the space $X_{\tau}$ defined by

$$
X_{\tau}=\left\{\left.\boldsymbol{v}_{\tau}\right|_{\Gamma_{3}}: \boldsymbol{v} \in V\right\}
$$

and we denote by $X_{\tau}^{\prime}$ its dual. We also consider the functional $J_{c}: X_{\tau}^{\prime} \times X_{\tau} \rightarrow \mathbb{R}$ defined by

$$
J_{c}\left(\boldsymbol{\gamma}, \boldsymbol{v}_{\tau}\right)=\left\langle\gamma, \boldsymbol{v}_{\tau}\right\rangle_{X_{\tau}^{\prime}, X_{\tau}}, \quad \forall \gamma \in X_{\tau}^{\prime}, \quad \forall \boldsymbol{v}_{\tau} \in X_{\tau},
$$

where $\langle\cdot, \cdot\rangle$ denotes the duality pairing between $X_{\tau}^{\prime}$ and $X_{\tau}$.

Proceeding in a standard way (see for instance $[17,18]$ ) and using the subdifferential inclusion $(7.1)$, we obtain the following variational formulation of the frictional Problem 5.1 in terms of velocity $\dot{\boldsymbol{u}}$ and stress multiplier $\lambda$.

Problem 7.1. Find a velocity field $\dot{\boldsymbol{u}}: \mathbb{R}_{+} \rightarrow V$ and a stress multiplier $\boldsymbol{\lambda}: \mathbb{R}_{+} \rightarrow X_{\tau}^{\prime}$ such that, for all $t \in \mathbb{R}_{+}$,

$$
\begin{aligned}
& (A \dot{\boldsymbol{u}}(t), \boldsymbol{v})_{V}+\varphi(\mathcal{S} \dot{\boldsymbol{u}}(t), \boldsymbol{v})+J_{c}\left(\boldsymbol{\lambda}(t), \boldsymbol{v}_{\tau}\right)=(\boldsymbol{f}(t), \boldsymbol{v})_{V} \quad \forall \boldsymbol{v} \in V, \\
& -\boldsymbol{\lambda}(t) \in g\left(\left\|\dot{\boldsymbol{u}}_{\tau}(t)\right\|\right) \partial\left\|\dot{\boldsymbol{u}}_{\tau}(t)\right\| \quad \text { in } \quad X_{\tau}^{\prime} .
\end{aligned}
$$

Using now (5.25), we obtain the following equivalent formulation of Problem 7.1, in terms of displacement $\boldsymbol{u}$ and stress multiplier $\boldsymbol{\lambda}$.

Problem 7.2. Find a displacement field $\boldsymbol{u}: \mathbb{R}_{+} \rightarrow V$ and a stress multiplier field $\boldsymbol{\lambda}: \mathbb{R}_{+} \rightarrow X_{\tau}^{\prime}$ such that, for all $t \in \mathbb{R}_{+}$,

$$
\begin{aligned}
& (A \dot{\boldsymbol{u}}(t), \boldsymbol{v})_{V}+\varphi(\boldsymbol{u}(t), \boldsymbol{v})+J_{c}\left(\boldsymbol{\lambda}(t), \boldsymbol{v}_{\tau}\right)=(\boldsymbol{f}(t), \boldsymbol{v})_{V} \quad \forall \boldsymbol{v} \in V, \\
& -\boldsymbol{\lambda}(t) \in g\left(\left\|\dot{\boldsymbol{u}}_{\tau}(t)\right\|\right) \partial\left\|\dot{\boldsymbol{u}}_{\tau}(t)\right\| \quad \text { in } \quad X_{\tau}^{\prime} .
\end{aligned}
$$

Next, we describe the numerical scheme for Problem 7.2 on a finite domain $[0, T] \times \Omega$. Let $0=t_{0}<t_{1}<$ $\ldots<t_{N}=T$ be a uniform partition of the time interval $[0, T]$, i.e., $t_{n}=n k, \quad 0 \leq n \leq N, k=T / N$. For a continuous function $f(t)$ we denote $f_{n}=f\left(t_{n}\right)$. In order to ensure the equivalence between the temporally 
discrete formulations issued from Problems 7.1 and 7.2 we use a middle point scheme for the discretization of the velocity,

$$
\delta \boldsymbol{u}_{n}=-\delta \boldsymbol{u}_{n-1}+\frac{2}{k}\left(\boldsymbol{u}_{n}-\boldsymbol{u}_{n-1}\right) \quad \forall n=1, \ldots, N,
$$

where $\delta \boldsymbol{u}_{n}$ denotes the discrete approximation of the velocity $\dot{\boldsymbol{u}}$ at the time $t_{n}$, for all $n=1, \ldots, N$. Also, we consider the approximation $\mathcal{S}_{n}^{k}$ of the operator $\mathcal{S}$ defined by

$$
\mathcal{S}_{n}^{k} \delta \boldsymbol{u}^{k}=k \sum_{j=0}^{n} \delta \boldsymbol{u}_{j}^{k}+\boldsymbol{u}_{0}, \quad \text { where } \quad \delta \boldsymbol{u}^{k}:=\left\{\delta \boldsymbol{u}_{n}^{k}\right\}_{n \geq 0} .
$$

Then, adding equations (7.7) for $n=1, \ldots, N$, we recover the trapezoidal rule (7.8), i.e.,

$$
\boldsymbol{u}_{n}=\mathcal{S}_{n}^{k} \delta \boldsymbol{u}_{n}=\frac{k}{2} \delta \boldsymbol{u}_{0}+k \delta \boldsymbol{u}_{1}+\ldots+k \delta \boldsymbol{u}_{n-1}+\frac{k}{2} \delta \boldsymbol{u}_{n}+\boldsymbol{u}_{0}
$$

We now briefly recall a fully discrete approximation in order to solve Problem 7.2. First, we consider a finite dimensional space $V^{h} \subset V$ approximating the spaces $V$, in which $h>0$ denotes the spatial discretization parameter. In the numerical simulations presented below the space $V^{h}$ consists of continuous and piecewise affine functions, that is,

$$
\begin{aligned}
V^{h}=\left\{\boldsymbol{v}^{h}\right. & \in[C(\bar{\Omega})]^{d}:\left.\boldsymbol{v}^{h}\right|_{T} \in\left[P_{1}(T)\right]^{d} \forall T \in \mathcal{T}^{h}, \\
\boldsymbol{v}^{h} & \left.=\mathbf{0} \text { at the nodes on } \Gamma_{1}, \quad v_{\nu}^{h}=0 \text { at the nodes on } \overline{\Gamma_{3}}\right\},
\end{aligned}
$$

where $\Omega$ is assumed to be a polyhedral domain, $\mathcal{T}^{h}$ denotes a finite element triangulation of $\bar{\Omega}$, and $P_{1}(T)$ represents the space of polynomials of degree less or equal to one in $T$. We also consider the discrete space $Y_{\tau}^{h} \subset X_{\tau}^{\prime} \cap L^{2}\left(\Gamma_{3}\right)$ related to the discretization of the stress $\boldsymbol{\lambda}$, see $[3,4,37]$ for details.

Using the middle point scheme (7.7) and some arguments in $[17,18]$, the fully discrete approximation of Problem 7.2 takes the following formulation, in which the discrete stress $\lambda_{n}^{h k}$ on the contact boundary $\Gamma_{3}$ can be viewed as a Lagrange stress multiplier.

Problem 7.3. Find a discrete displacement field $\boldsymbol{u}^{h k}=\left\{\boldsymbol{u}_{n}^{h k}\right\}_{n=0}^{N} \subset V^{h}$ and a discrete stress multiplier field $\boldsymbol{\lambda}^{h k}=\left\{\boldsymbol{\lambda}_{n}^{h k}\right\}_{n=0}^{N} \subset Y_{\tau}^{h}$ such that, for all $n=1, \ldots, N$,

$$
\begin{aligned}
& \left(A \delta \boldsymbol{u}_{n}^{h k}, \boldsymbol{v}^{h}\right)_{V}+\varphi\left(\boldsymbol{u}_{n}^{h k}, \boldsymbol{v}^{h}\right)+J_{c}\left(\boldsymbol{\lambda}_{n}^{h k}, \boldsymbol{v}_{\tau}^{h}\right)=\left(\boldsymbol{f}_{n}, \boldsymbol{v}^{h}\right)_{V} \quad \forall \boldsymbol{v}^{h} \in V^{h}, \\
& -\boldsymbol{\lambda}_{n}^{h k} \in g\left(\left\|\left(\delta \boldsymbol{u}_{\tau}\right)_{n}^{h k}\right\|\right) \partial\left\|\left(\delta \boldsymbol{u}_{\tau}\right)_{n}^{h k}\right\| \quad \text { on } \quad \Gamma_{3} .
\end{aligned}
$$

Here $\boldsymbol{u}_{0}^{h k}$ and $\delta \boldsymbol{u}_{0}^{h k}$ are appropriate approximations of the initial conditions $\boldsymbol{u}_{0}$ and $\delta \boldsymbol{u}_{0}$, see (7.7). In the rest of the section, to simplify the notation, we skip the dependence of various variables with respect to the discretization parameters $n, k$ and $h$, i.e., for example, we write $\boldsymbol{u}$ instead of $\boldsymbol{u}_{n}^{h k}$. For each incremental time step $n$, the numerical solution of the system (7.10)-(7.11) is based on the following iterative algorithm, in which $q$ represents the index of the iterative procedure.

Problem 7.4. Let $\epsilon>0$ and $\boldsymbol{u}^{(0)}$ be given. Then, for $q=0,1 \ldots$, find a displacement field $\boldsymbol{u}^{(q+1)} \in V^{h}$ and a stress multiplier $\boldsymbol{\lambda}^{(q+1)} \in Y_{\tau}^{h}$ such that

$$
\begin{aligned}
& (A \delta \boldsymbol{u}, \boldsymbol{v})_{V}+\varphi(\boldsymbol{u}, \boldsymbol{v})+J_{c}\left(\boldsymbol{\lambda}^{(q+1)}, \boldsymbol{v}_{\tau}\right)=(\boldsymbol{f}, \boldsymbol{v})_{V} \quad \forall \boldsymbol{v} \in V^{h}, \\
& \boldsymbol{\lambda}^{(q+1)} \in-g\left(\left\|\delta \boldsymbol{u}_{\tau}^{(q)}\right\|\right) \partial\left\|\delta \boldsymbol{u}_{\tau}^{(q+1)}\right\| \quad \text { on } \quad \Gamma_{3},
\end{aligned}
$$

until

$$
\left\|\boldsymbol{u}^{(q+1)}-\boldsymbol{u}^{(q)}\right\|_{V^{h}} \leq \epsilon\left\|\boldsymbol{u}^{(q)}\right\|_{V^{h}} \text { and }\left\|\boldsymbol{\lambda}^{(q+1)}-\boldsymbol{\lambda}^{(q)}\right\|_{Y_{\tau}^{h}} \leq \epsilon\left\|\boldsymbol{\lambda}^{(q)}\right\|_{Y_{\tau}^{h}} .
$$




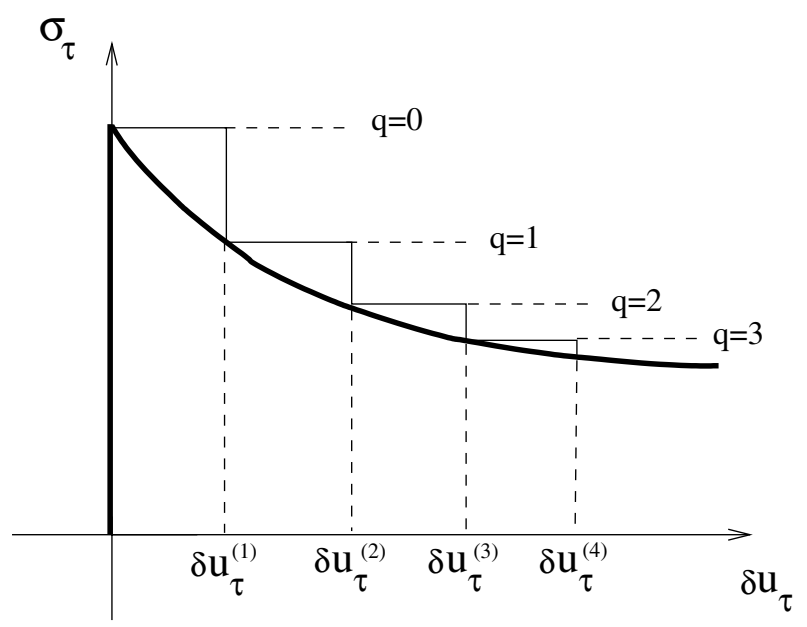

FiguRE 1. Graphical description of the iterative algorithm.

This numerical strategy is depicted in Figure 1. Note that it is based on an iterative procedure in which, at each iteration, the friction bound $g$ is fixed to a constant value which depends on the tangential velocity solution $\delta \boldsymbol{u}_{\tau}^{(q)}$ found in the previous iteration $q$. Then, the resulting iterative problems can be solved by classical numerical methods. Different numerical methods in the study of such problems with nonmonotone friction laws, including the proximal bundle methods, can be found in [15,24,25,35].

In order to keep this paper in a reasonable length, we skip the description of the numerical algorithm used to solve the system (7.12)-(7.13). We only comment that the numerical treatment of this system is based on an augmented Lagrangian approach and on a linear iterative method similar to that used in the Newton method, see [1] for details. To this end we consider additional fictitious nodes for the Lagrange multiplier in the initial mesh. The construction of these nodes depends on the contact element used for the geometrical discretization of the interface $\Gamma_{3}$. In the case of the numerical example presented below, the discretization is based on "nodeto-rigid" contact element, which is composed by one node of $\Gamma_{3}$ and one Lagrange multiplier node. Details on Computational Contact Mechanics, including algorithms similar to that used above can be found in the monographs $[22,37]$.

Numerical example. We consider the physical setting depicted in Figure 2 . There, $\Omega=\left(0, L_{1}\right) \times\left(0, L_{2}\right) \subset \mathbb{R}^{2}$ with $L_{1}, L_{2}>0$ and

$$
\Gamma_{1}=\{0\} \times\left[0, L_{2}\right], \Gamma_{2}=\left(\left\{L_{1}\right\} \times\left[0, L_{2}\right]\right) \cup\left(\left[0, L_{1}\right] \times\left\{L_{2}\right\}\right), \Gamma_{3}=\left[0, L_{1}\right] \times\{0\} .
$$

The domain $\Omega$ represents the cross section of a three-dimensional linearly viscoelastic body subjected to the action of tractions in such a way that a plane stress hypothesis is assumed. On the part $\Gamma_{1}=\{0\} \times\left[0, L_{2}\right]$ the body is clamped and, therefore, the displacement field vanishes there. Vertical tractions act on the part $\left[0, L_{1}\right] \times\left\{L_{2}\right\}$ of the boundary and the part $\left\{L_{1}\right\} \times\left[0, L_{2}\right]$ is traction free. No body forces are assumed to act on the viscoelastic body during the process. The body is in bilateral frictional contact with a rigid obstacle on the part $\Gamma_{3}=\left[0, L_{1}\right] \times\{0\}$ of the boundary. The friction is modeled by a nonmonotone version of Coulomb's law in which the friction bound $g$ is given by

$$
g\left(\left\|\dot{\boldsymbol{u}}_{\tau}\right\|\right)=(a-b) \times \mathrm{e}^{-\alpha\left\|\dot{\boldsymbol{u}}_{\tau}\right\|}+b,
$$

with $a, b, \alpha>0, a \geq b$. Note that the friction law (5.6) with the friction bound (7.14) describes the slip weakening phenomenon which appears in the study of geophysical problems, see [34]. Indeed, in this case the friction bound 


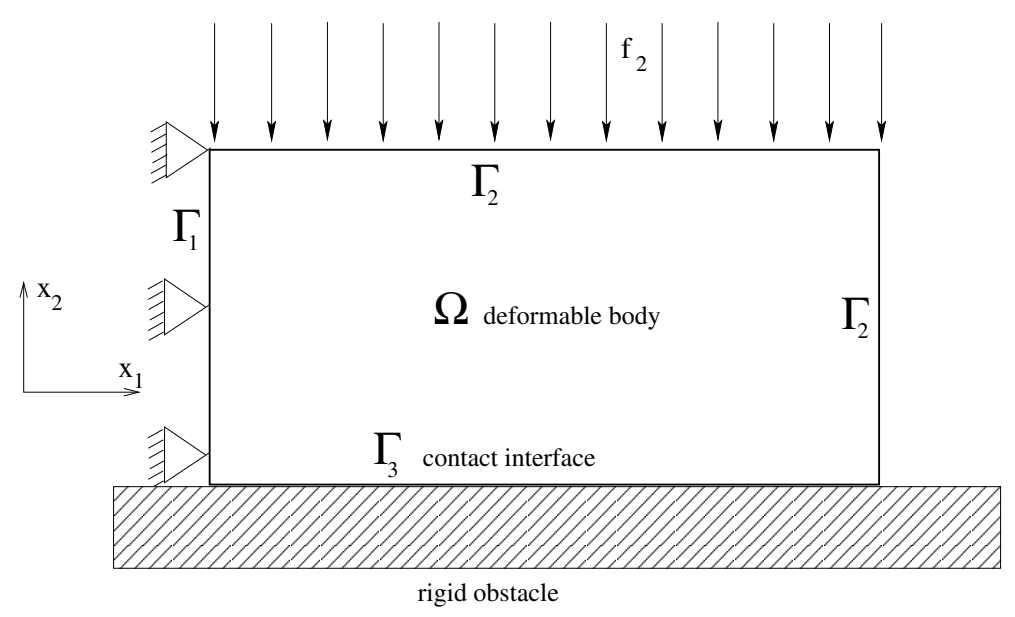

Figure 2. Initial configuration of the two-dimensional example.

decreases with the slip-rate from the value $a$ to the limit value $b$. And, for this reason, this friction law can be characterized as being nonmonotone.

The material behavior is governed with a viscoelastic linear constitutive law defined by the viscosity tensor $\mathcal{A}$ and the elasticity tensor $\mathcal{B}$ given by

$$
\begin{aligned}
& (\mathcal{A} \boldsymbol{\tau})_{\alpha \beta}=\mu_{1}\left(\tau_{11}+\tau_{22}\right) \delta_{\alpha \beta}+\mu_{2} \tau_{\alpha \beta}, \quad 1 \leq \alpha, \beta \leq 2, \forall \boldsymbol{\tau} \in \mathbb{S}^{2}, \\
& (\mathcal{B} \boldsymbol{\tau})_{\alpha \beta}=\frac{E \kappa}{(1-\kappa)(1-2 \kappa)}\left(\tau_{11}+\tau_{22}\right) \delta_{\alpha \beta}+\frac{E}{1+\kappa} \tau_{\alpha \beta}, \quad 1 \leq \alpha, \beta \leq 2, \forall \boldsymbol{\tau} \in \mathbb{S}^{2},
\end{aligned}
$$

where $\mu_{1}$ and $\mu_{2}$ are viscosity constants, $E$ and $\kappa$ are Young's modulus and Poisson's ratio of the material, and $\delta_{\alpha \beta}$ denotes the Kronecker delta symbol.

For computation we use the following data:

$$
\begin{aligned}
& L_{1}=1 \mathrm{~m}, \quad L_{2}=0.5 \mathrm{~m}, \quad T=1, \\
& \boldsymbol{u}_{0}=0 \mathrm{~m}, \quad \delta \boldsymbol{u}_{0}=0 \mathrm{~m} / \mathrm{s}, \\
& \mu_{1}=0.05 \mathrm{~N} / \mathrm{m}, \quad \mu_{2}=0.1 \mathrm{~N} / \mathrm{m}, \quad E=1 \mathrm{~N} / \mathrm{m}, \quad \kappa=0.3, \\
& \boldsymbol{f}_{0}=(0,0) \mathrm{N} / \mathrm{m}^{2}, \quad \boldsymbol{f}_{2}=\left\{\begin{array}{cc}
(0,0) \mathrm{N} / \mathrm{m} & \text { on }\{1\} \times[0,0.5], \\
(0,-0.3) \mathrm{N} / \mathrm{m} & \text { on }[0,1] \times\{0.5\},
\end{array}\right. \\
& a=0.003, \quad b=0.001, \quad \alpha=100, \quad \epsilon=10^{-6} .
\end{aligned}
$$

Error estimates. In order to see the convergence behavior of the fully discrete scheme, we compute a sequence of numerical solutions by using uniform partitions of the time interval $[0,1]$ and uniform triangulations of the body. For instance, for $h=1 / 32, k=1 / 32$ and $N=32$ we obtain the deformed configuration and the contact interface forces plotted in Figure 3.

The numerical solution is obtained by considering Problem 7.2 in which we provide the estimated error values for several discretization parameters $h$ and $k$ in the form

$$
\max _{1 \leq n \leq N}\left\{\left\|\boldsymbol{u}_{n}-\boldsymbol{u}_{n}^{h k}\right\|_{V}\right\}
$$




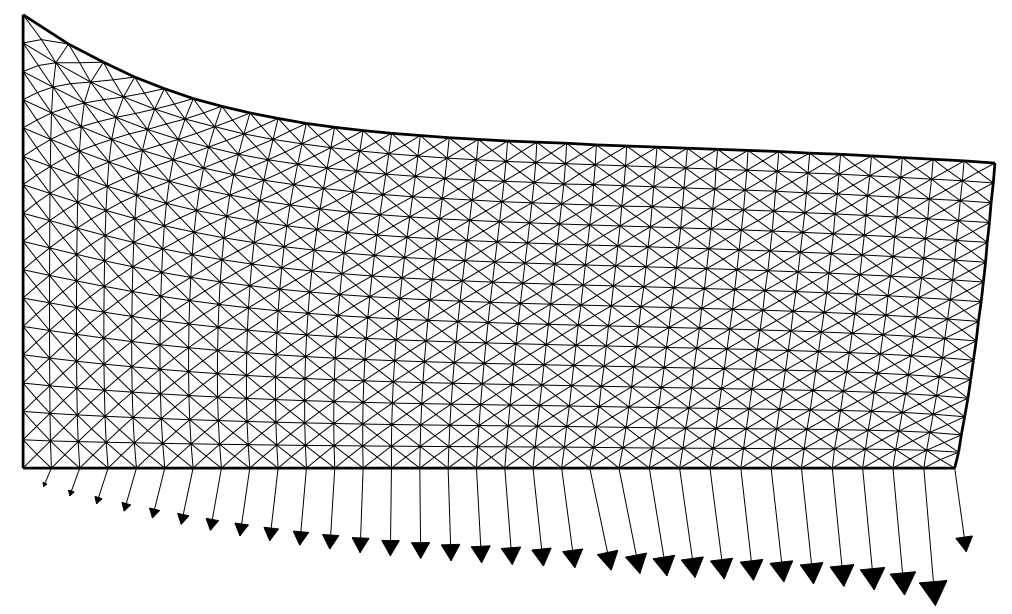

FiguRE 3. Deformed mesh and contact interface forces on $\Gamma_{3}$.

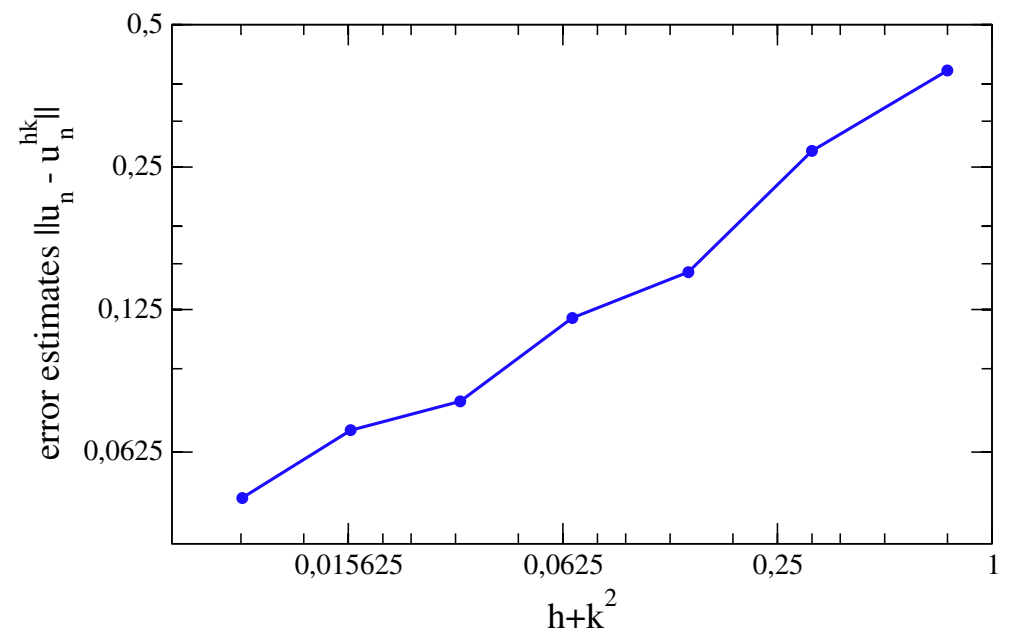

FIGURE 4. Estimated numerical errors.

Here, the boundary $\Gamma$ of $\Omega$ is divided into $1 / h$ equal parts. We start with $h=1 / 2$ and $k=1 / 2$ which are successively halved. The numerical solution corresponding to $h=1 / 256$ and $k=1 / 256$ is taken as the "exact" solution, which is used to compute the errors of the numerical solutions with larger values of $h$ and $k$; this discretization corresponds to a problem with 132354 degrees of freedom. The numerical results are presented in Figure 4 where the dependence of the error estimate $\left\|\boldsymbol{u}_{n}-\boldsymbol{u}_{n}^{h k}\right\|_{V}$ with respect to $h+k^{2}$ is plotted. Note that these results validate the theoretic estimate obtained in Section 6.

Monotonicity and stick-slip phenomenon. In the rest of this section, we present more results concerning the behavior of our model of friction. The tangential stresses and the tangential velocities on $\Gamma_{3}$ are presented in Figures 5 and 6, respectively. In each of these figures, three curves are plotted, corresponding to different values of the coefficients $a$ and $b$. It is easy to see that the case $a=0.003$ and $b=0.001$ lead to a nonmonotone behavior of the tangential stress with respect to the tangential velocity on $\Gamma_{3}$ while the cases $a=b=0.003$ and $a=b=0.001$ correspond to the classical monotone Coulomb's law of dry friction. In Figure 7, we plot graph of the tangential stress versus tangential velocity at the node of coordinates $(1,0)$ on $\Gamma_{3}$, for each incremental time 


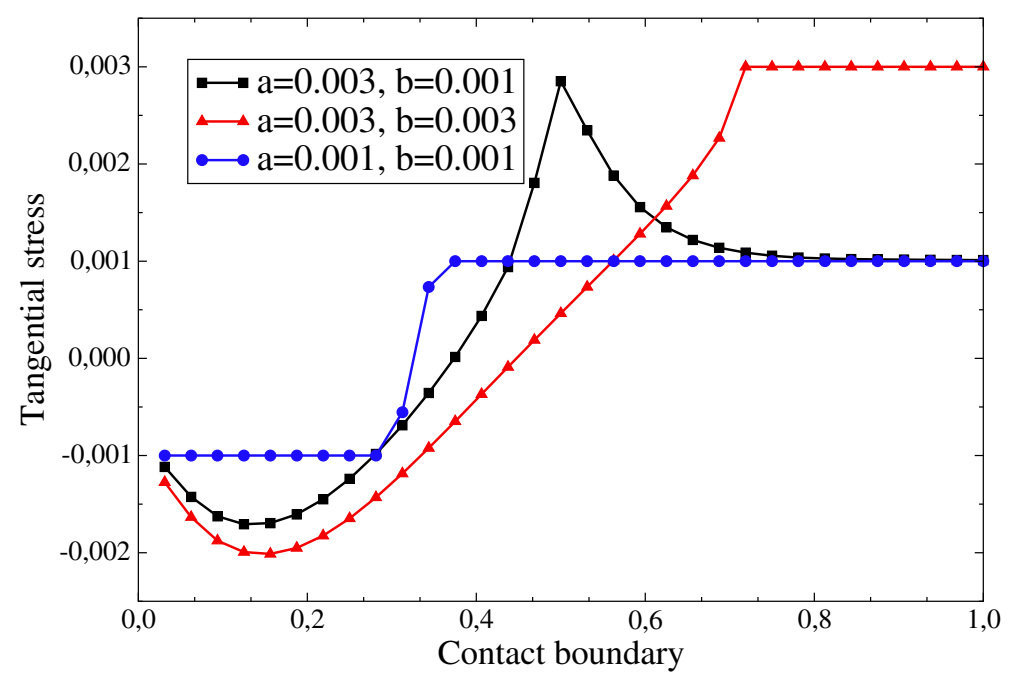

Figure 5. Tangential stresses on $\Gamma_{3}$.

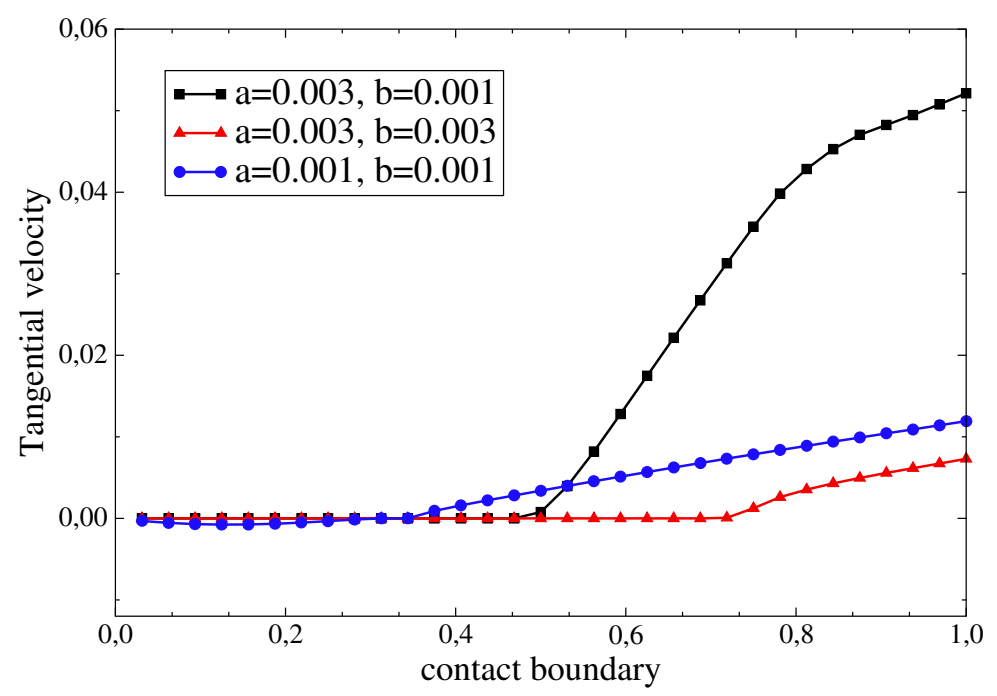

Figure 6. Tangential velocities on $\Gamma_{3}$.

step $n$ of Problem 7.3. Moreover, in the case $a=0.003$ and $b=0.001$, we plot the numerical solution obtained at each iteration of the algorithm presented in Problem 7.4, during each increment of time. This representation reflects a stick-slip phenomenon. First, we observe a stick behavior characterized by the segment $O A$, next a slip behavior appears with the decreasing evolution between the points $A$ and $B$. And, we pass from point $B$ to point $C$ to recover a stick behavior on the segment $C D$. Finally, the evolution between $D$ and $E$ corresponds again to a slip behavior.

Detail of the computations for these mechanical results is the following. The problem is discretized in 2048 finite elements and 32 contact elements $(h=1 / 32)$ with a time step $k=1 / 32$; the total number of degrees of freedom is equal to 2210. In the case of simulations presented in Figure 7, we consider only 10 time incremental steps in order to obtain a better visualization of the solutions of Problems 7.3 and 7.4. 


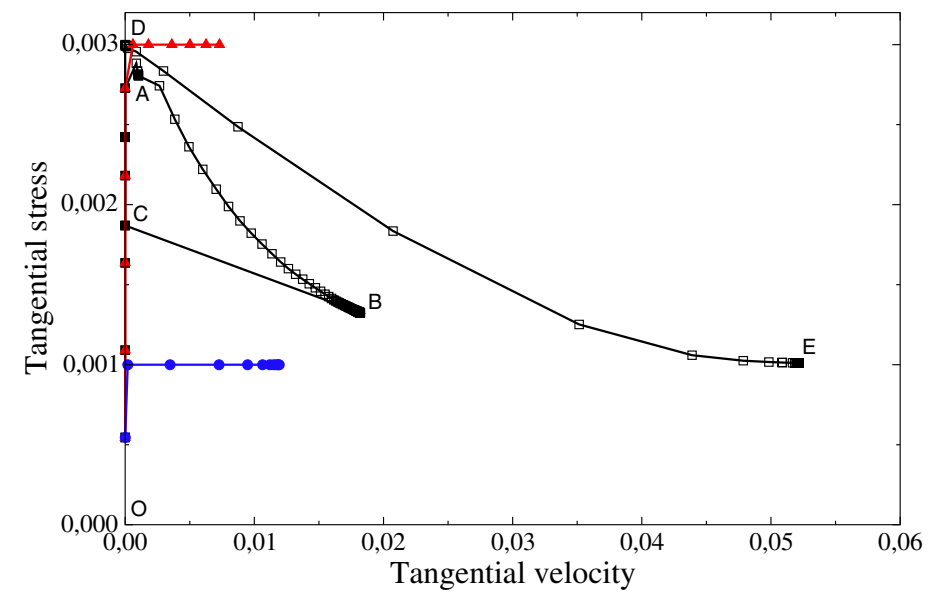

- $\mathrm{a}=0.003, \mathrm{~b}=0.001$ (solutions at each time increment of Problem 12)

$\square \mathrm{a}=0.003, \mathrm{~b}=0.001$ (solutions at each iteration of Problem 13)

$\longleftrightarrow \mathrm{a}=0.003, \mathrm{~b}=0.003$

$\bullet a=0.001, b=0.001$

FiguRE 7. Tangential stresses versus tangential velocities at the node of coordinates $(1,0)$ on $\Gamma_{3}$ for each time incremental step of Problem 7.3.

For information, with $h=1 / 32$ and $k=1 / 32$, the simulation runs in 49 CPU time (expressed in seconds). In the nonmonotone case, the average number of iterations $q$ per time step for the solution of system (7.10)(7.11) is equal to 7.2. The computations were realized on an IBM Blade Center $\mathrm{H}$ computer server of the Computing Center of the University of Perpignan, France. The computer server consists of one master IBM node (Intel Woodcrest processor, Model 5148) and 14 computer IBM nodes (Inter Dual core processor, Model 5148, $2.33 \mathrm{GHz})$.

Acknowledgements. This research was supported by a Marie Curie International Research Staff Exchange Scheme Fellowship within the 7th European Community Framework Programme under Grant Agreement no. PIRSES-GA-2011-295118.

\section{REFERENCES}

[1] P. Alart and A. Curnier, A mixed formulation for frictional contact problems prone to Newton like solution methods. Comput. Methods Appl. Mech. Eng. 92 (1991) 353-375.

[2] C. Baiocchi and A. Capelo, Variational and Quasivariational Inequalities: Applications to Free-Boundary Problems. John Wiley, Chichester (1984).

[3] M. Barboteu and M. Sofonea, Modelling and analysis of the unilateral contact of a piezoelectric body with a conductive support. J. Math. Anal. Appl. 358 (2009) 110-124.

[4] M. Barboteu and M. Sofonea, Analysis and numerical approach of a piezoelectric contact problem. Annals of the Academy of Romanian Scientists, Series on Mathematics and its Applications 1 (2009) 7-31.

[5] D. Braess, Finite Elements: Theory, Fast Solvers, and Applications in Solid Mechanics, 3rd edn. Cambridge University Press, Cambridge (2007).

[6] S.C. Brenner and L.R. Scott, The Mathematical Theory of Finite Element Methods, 3rd edn. Springer-Verlag, New York (2008).

[7] H. Brezis, Equations et inéquations non linéaires dans les espaces vectoriels en dualité. Ann. Inst. Fourier 18 (1968) 115-175.

[8] P.G. Ciarlet, Basic error estimates for elliptic problems, in Handbook of Numerical Analysis, vol. II, edited by P.G. Ciarlet and J.-L. Lions. North-Holland, Amsterdam (1991) 17-351.

[9] G. Duvaut and J.L. Lions, Inequalities in Mechanics and Physics. Springer-Verlag, Berlin (1976).

[10] R. Glowinski, Numerical Methods for Nonlinear Variational Problems. Springer-Verlag, New York (1984).

[11] C. Eck, J. Jarušek and M. Krbeč, Unilateral Contact Problems: Variational Methods and Existence Theorems, vol. 270, Pure Appl. Math. Chapman/CRC Press, New York (2005). 
[12] W. Han and B.D. Reddy, Computational plasticity: the variational basis and numerical analysis. Comput. Mech. Adv. 2 (1995) $283-400$.

[13] W. Han and B.D. Reddy, Plasticity: Mathematical Theory and Numerical Analysis, 2nd edn. Springer-Verlag, New York (2013).

[14] W. Han and M. Sofonea, Quasistatic Contact Problems in Viscoelasticity and Viscoplasticity. In vol. 30, Stud. Adv. Math. American Mathematical Society, Providence, RI-International Press, Sommerville, MA (2002).

[15] J. Haslinger, M. Miettinen and P.D. Panagiotopoulos, Finite Element Method for Hemivariational Inequalities. Theory, Methods Appl. Kluwer Academic Publishers, Boston, Dordrecht, London (1999).

[16] I. Hlaváček, J. Haslinger, J. Necăs and J. Lovíšek, Solution of Variational Inequalities in Mechanics. Springer-Verlag, New York (1988).

[17] H.B. Khenous, P. Laborde, and Y. Renard, On the discretization of contact problems in elastodynamics. Lect. Notes Appl. Comput. Mech. 27 (2006) 31-38.

[18] H.B. Khenous, J. Pommier and Y. Renard, Hybrid discretization of the Signorini problem with Coulomb friction. Theoretical aspects and comparison of some numerical solvers. Appl. Numer. Math. 56 (2006) 163-192.

[19] N. Kikuchi and J.T. Oden, Theory of variational inequalities with applications to problems of flow through porous media. Int. J. Engng. Sci. 18 (1980) 1173-1284.

[20] N. Kikuchi and T.J. Oden, Contact Problems in Elasticity. SIAM, Philadelphia (1988).

[21] D. Kinderlehrer and G. Stampacchia, An Introduction to Variational Inequalities and their Applications. In vol. 31, Classics Appl. Math. SIAM, Philadelphia (2000).

[22] T. Laursen, Computational contact and impact mechanics. Springer, Berlin (2002).

[23] J.A.C. Martins and M.D.P. Monteiro Marques, eds., Contact Mechanics. Kluwer, Dordrecht (2002).

[24] E.S. Mistakidis and P.D. Panagiotopulos, Numerical treatment of problems involving nonmonotone boundary or stress-strain laws. Comput. Structures 64 (1997) 553-565.

[25] E.S. Mistakidis and P.D. Panagiotopulos, The search for substationary points in the unilateral contact problems with nonmonotone friction. Math. Comput. Modelling 28 (1998) 341-358.

[26] P.D. Panagiotopoulos, Inequality Problems in Mechanics and Applications. Birkhäuser, Boston, 1985.

[27] M. Raous, M. Jean and J.J. Moreau, Contact Mechanics. Plenum Press, New York (1995).

[28] M. Shillor, ed., Recent advances in contact mechanics, Special issue of Math. Comput. Modelling 28 (4-8) (1998).

[29] M. Shillor, M. Sofonea and J.J. Telega, Models and Analysis of Quasistatic Contact. Variational Methods. In vol. 655, Lect. Notes Phys. Springer, Berlin (2004).

[30] M. Sofonea, C. Avramescu and A. Matei, A fixed point result with applications in the study of viscoplastic frictionless contact problems. Commun. Pure Appl. Anal. 7 (2008) 645-658.

[31] M. Sofonea, W. Han and M. Shillor, Analysis and Approximation of Contact Problems with Adhesion or Damage. Chapman \& Hall/CRC, New York (2006).

[32] M. Sofonea and A. Matei, Variational Inequalities with Applications. A Study of Antiplane Frictional Contact Problems. vol. 18, Adv. Mech. Math. Springer, New York (2009).

[33] M. Sofonea and A. Matei, History-dependent quasivariational inequalities arising in contact mechanics. Eur. J. Appl. Math. 22 (2011) 471-491.

[34] C.H. Scholz, The Mechanics of Earthquakes and Faulting. Cambridge University Press (1990).

[35] M.A. Tzaferopoulos, E.S. Mistakidis, C.D. Bisbos, and P.D. Panagiotopulos, Comparison of two methods for the solution of a class of nonconvex energy problems using convex minimization algorithms. Comput. Struct. 57 (1995) 959-971.

[36] P. Wriggers and U. Nackenhorst, eds., Analysis and Simulation of Contact Problems. In vol. 27, Lect. Notes Appl. Comput. Mech. Springer, Berlin (2006).

[37] P. Wriggers, Computational Contact Mechanics. Wiley, Chichester (2002). 